\title{
MEASURES WITH BOUNDED POWERS ON LOCALLY COMPACT ABELIAN GROUPS
}

\author{
BY
}

G. V. WOOD

\begin{abstract}
If $\mu$ is a measure on a locally compact abelian group with its positive and negative convolution powers bounded in norm by $K<\frac{1}{3}(4 \cos (\pi / 9)+1) \sim$ 1.58626, then $\mu$ has the form $\mu=\lambda\left(\cos \theta \delta_{x}+i \sin \theta \delta_{x u}\right)$ where $|\lambda|=1$ and $u^{2}=e$. Applications to isomorphism theorems are given. In particular, if $G_{1}$ and $G_{2}$ are 1.c.a. groups and $T$ is an isomorphism of $L^{1}\left(G_{1}\right)$ onto $L^{1}\left(G_{2}\right)$ with $\|T\|$ $<\frac{1}{3}(4 \cos (\pi / 9)+1)$, then either $G_{1}$ and $G_{2}$ are isomorphic, or they both have subgroups of order 2 with isomorphic quotients.
\end{abstract}

1. Introduction. In this paper, we characterize all measures on a locally compact abelian group that have the norms of their (positive and negative) convolution powers bounded by a number $K<\frac{1}{3}(4 \cos (\pi / 9)+1) \sim 1.58626$. In fact, such measures are supported on a coset of a subgroup of order 2 and have the form

$$
\mu=\lambda\left(\cos \theta \delta_{x}+i \sin \theta \delta_{x u}\right)
$$

where $u^{2}=e$ and $|\lambda|=1$. These measures actually have the norms of their convolution powers bounded by $\sqrt{ } 2$. The result is the best possible in the sense that there is a measure supported on a group of order 3 which has its powers bounded in norm by $\frac{1}{3}(4 \cos (\pi / 9)+1)$. For example

$$
\mu=\frac{1}{3}\left(1+2 \cos \frac{2 \pi}{9}\right) \delta_{e}+\frac{1}{3}\left(1+2 \cos \frac{8 \pi}{9}\right) \delta_{u}+\frac{1}{3}\left(1+2 \cos \frac{14 \pi}{9}\right) \delta_{u^{2}}
$$

where $u^{3}=e$.

This paper may be thought of as a continuation-and improvement-of [3]. Characterizing measures with powers bounded in norm by $K$ is equivalent to the problem of characterizing homomorphisms between group algebras with norm less than $K$. If $T: l_{1}\left(G_{1}\right) \rightarrow l_{1}\left(G_{2}\right)$ is a homomorphism with $\|T\| \leqslant K$, then for $x \in G_{1}$, $T x \in l_{1}\left(G_{2}\right)$ is a measure with powers norm-bounded by $K$. Conversely, if $\mu$ is a measure on $G$ with powers norm-bounded by $K$, then there is a homomorphism of $l_{1}(\mathrm{Z})$ into $l_{1}(G)$ with norm less than $K$ defined by

$$
T\left(\sum_{-\infty}^{\infty} \lambda_{n} \delta_{n}\right)=\sum_{n=-\infty}^{\infty} \lambda_{n} \mu^{n}
$$

Received by the editors February 20, 1980 and, in revised form, July 31, 1980 and October 9, 1980. 1980 Mathematics Subject Classification. Primary 43A10, 43A05; Secondary 43A22, 43A20.

Key words and phrases. Measures, bounded powers, locally compact abelian groups, algebra, homomorphisms, algebra isomorphisms. 
Thus in [3], it is implicit that measures with powers norm-bounded by $K<\sqrt{ } 2$ have the form (1) with $\theta=2 \pi / n$ for an odd number $n$. The methods used there did not give a result even for $K=\vee 2$.

Here, we show that when $K=\sqrt{ } 2$, then measures have the form (1) without restriction on $\theta$. We also show that no measures exist where the precise bound is between $\sqrt{ } 2$ and $\frac{1}{3}(4 \cos (\pi / 9)+1)$.

In [3], it was shown that there is an algebra isomorphism of $l_{1}\left(\mathbf{Z}_{2} \times \mathbf{Z}_{2}\right)$ onto $l_{1}\left(\mathbf{Z}_{4}\right)$ with norm $\vee 2$. This occurs because the two groups concerned each have a subgroup of order 2 and their quotients are isomorphic. In $\S 3$, we show that if $T$ is an algebra isomorphism of $l_{1}\left(G_{1}\right)$ onto $l_{1}\left(G_{2}\right)$ with norm less than

$$
\frac{1}{3}(4 \cos (\pi / 9)+1)
$$

then either $G_{1}$ and $G_{2}$ are isomorphic, or they each have a subgroup of order 2 and their quotients are isomorphic. In particular, if the groups have no elements of order 2 , then $G_{1}$ and $G_{2}$ must be isomorphic. This last result is best possible, since there is an isomorphism of $l_{1}\left(\mathbf{Z}_{3} \times \mathbf{Z}_{3}\right)$ onto $l_{1}\left(\mathbf{Z}_{9}\right)$ with norm precisely

$$
\frac{1}{3}(4 \cos (\pi / 9)+1)
$$

(See Example in §3.)

The general method is similar to that contained in [3], but the estimates here have to be made much more carefully. Sometimes the estimates are incredibly close-a pocket calculator is essential! It is perhaps surprising that the characterization of measures with bounded powers in [5] and homomorphisms in [4] seem to be no help in proving these quantitative results.

The first lemma gives the estimates which arise from an $l_{1}$-sequence with norm $K$ and $l_{2}$-norm equal to 1 . No properties of groups are used, and these are the estimates that provide the improvement to [3].

LEMMA 1. If $\left(\alpha_{i}\right) \in l_{1}$ with $\sum\left|\alpha_{i}\right|=K$ and $\sum\left|\alpha_{i}\right|^{2}=1$, then if $\left|\alpha_{1}\right| \geqslant\left|\alpha_{2}\right| \geqslant\left|\alpha_{3}\right|$

(a)

$$
\left|\alpha_{1}\right| \geqslant \max \left\{\frac{1}{K}, \frac{K}{3}+\frac{1}{3} \sqrt{ }\left(\frac{3-K^{2}}{2}\right)\right\},
$$

(b)

$$
\left|\alpha_{2}\right| \geqslant \frac{1-\left|\alpha_{1}\right|^{2}}{K-\left|\alpha_{1}\right|}
$$

(c)

$$
\left|\alpha_{2}\right| \geqslant \frac{1}{2}\left(K-\left|\alpha_{1}\right|\right)+\sqrt{ }\left\{\frac{1}{2}\left(1-\left|\alpha_{1}\right|^{2}\right)-\frac{1}{4}\left(K-\left|\alpha_{1}\right|\right)^{2}\right\}
$$

whenever the expression under the square root is positive i.e. when

$$
\left|\alpha_{1}\right| \leqslant \frac{1}{3} K+\frac{2}{3} \sqrt{ }\left(\frac{3-K^{2}}{2}\right)
$$


(d)

$$
\left|\alpha_{3}\right|<\frac{1}{3} K-\frac{1}{3} \sqrt{ }\left(\frac{3-K^{2}}{2}\right) .
$$

In particular, if $K<\frac{1}{3}(4 \cos (\pi / 9)+1)$,

(e) $\left|\alpha_{1}\right|>0.69269$,

(f) $\left|\alpha_{3}\right|<0.36481$.

Proof. The first part of (a) is simply the inequality

$$
\left(\max _{i}\left|\alpha_{i}\right|\right)\left(\sum_{i=1}^{\infty}\left|\alpha_{i}\right|\right)>\sum_{i=1}^{\infty}\left|\alpha_{i}\right|^{2}=1
$$

while (b) is

$$
\left(\max _{i>2}\left|\alpha_{i}\right|\right)\left(\sum_{i=2}^{\infty}\left|\alpha_{i}\right|\right) \geqslant \sum_{i=2}^{\infty}\left|\alpha_{i}\right|^{2}
$$

For (c), we have

$$
\sum_{i=3}^{\infty}\left|\alpha_{i}\right|^{2}<\left(\sum_{i=3}^{\infty}\left|\alpha_{i}\right|\right)^{2}
$$

which is the same as

$$
1-\left|\alpha_{1}\right|^{2}-\left|\alpha_{2}\right|^{2}<\left(K-\left|\alpha_{1}\right|-\left|\alpha_{2}\right|\right)^{2} .
$$

Rearranging this as a condition on $\left|\alpha_{2}\right|$ gives (c). The second part of (a) is obtained by putting $\left|\alpha_{2}\right|<\left|\alpha_{1}\right|$ in inequality (c), and solving for $\left|\alpha_{1}\right|$.

For (d), consider the following two cases.

(1) If

$$
\left|\alpha_{1}\right|<\frac{1}{3} K+\frac{2}{3} \sqrt{ }\left(\frac{3-K^{2}}{2}\right),
$$

then by (c) we have

$$
\left|\alpha_{1}\right|+\left|\alpha_{2}\right|>\frac{1}{2}\left(K+\left|\alpha_{1}\right|\right)+\sqrt{ }\left\{\frac{1}{2}\left(1-\left|\alpha_{1}\right|^{2}\right)-\frac{1}{4}\left(K-\left|\alpha_{1}\right|\right)^{2}\right\}
$$

which decreases as $\left|\alpha_{1}\right|$ increases, since the derivative with respect to $\left|\alpha_{1}\right|$ is

$$
\frac{1}{2}-\frac{1}{4}\left(3\left|\alpha_{1}\right|-K\right)\left\{\frac{1}{2}\left(1-\left|\alpha_{1}\right|^{2}\right)-\frac{1}{4}\left(K-\left|\alpha_{1}\right|\right)^{2}\right\}^{-1 / 2}
$$

which is negative since

$$
\left|\alpha_{1}\right|>\frac{1}{3} K+\frac{1}{3} \sqrt{ }\left(\frac{3-K^{2}}{2}\right)
$$

by (a). Thus

$$
\begin{aligned}
\left|\alpha_{1}\right|+\left|\alpha_{2}\right| & >\frac{1}{2} K+\frac{1}{2}\left\{\frac{1}{3} K+\frac{2}{3} \sqrt{ }\left(\frac{3-K^{2}}{2}\right)\right\} \\
& =\frac{2}{3} K+\frac{1}{3} \sqrt{ }\left(\frac{3-K^{2}}{2}\right) .
\end{aligned}
$$


Now

(2) If

$$
\left|\alpha_{3}\right| \leqslant K-\left|\alpha_{1}\right|-\left|\alpha_{2}\right| \leqslant \frac{1}{3} K-\frac{1}{3} \sqrt{ }\left(\frac{3-K^{2}}{2}\right)
$$

$$
\left|\alpha_{1}\right|>\frac{1}{3} K+\frac{2}{3} \sqrt{ }\left(\frac{3-K^{2}}{2}\right)
$$

then we have

$$
\begin{aligned}
\left|\alpha_{3}\right| & \leqslant \sqrt{ } \frac{1}{2}\left\{1-\left|\alpha_{1}\right|^{2}\right\} \leqslant \sqrt{ } \frac{1}{2}\left\{1-\left(\frac{1}{3} K+\frac{2}{3} \sqrt{ }\left(\frac{3-K^{2}}{2}\right)\right)^{2}\right\} \\
& =\sqrt{ } \frac{1}{2}\left\{1-\frac{1}{9} K^{2}-\frac{4}{9} K \sqrt{ }\left(\frac{3-K^{2}}{2}\right)-\frac{4}{9}\left(\frac{3-K^{2}}{2}\right)\right\} \\
& =\sqrt{ } \frac{1}{2}\left\{\frac{1}{3}+\frac{1}{9} K^{2}-\frac{4}{9} K \sqrt{ }\left(\frac{3-K^{2}}{2}\right)\right\}=\frac{1}{3} K-\frac{1}{3} \sqrt{ }\left(\frac{3-K^{2}}{2}\right)
\end{aligned}
$$

Thus (d) is proved, and (e) and (f) follows from (a) and (d) by substitution. This completes the lemma.

Note that $\left|\alpha_{1}\right|+\left|\alpha_{2}\right| \geqslant \min \{1,2 / K\}$. For, by Lemma 1 (b) $\left|\alpha_{1}\right|+\left|\alpha_{2}\right| \geqslant\left|\alpha_{1}\right|+$ $\left(\left(1-\left|\alpha_{1}\right|^{2}\right) /\left(K-\left|\alpha_{1}\right|\right)\right)$ which is a convex function of $\left|\alpha_{1}\right|$. Hence, since $1 / K \leqslant$ $\left|\alpha_{1}\right| \leqslant 1,\left|\alpha_{1}\right|+\left|\alpha_{2}\right| \geqslant \min \{1,2 / K\}$.

2. Measures with bounded powers. Let $G$ be an abelian group. For most of this section we will not be concerned with any topological property of the group. In $l_{1}(G)$, the convolution of $\mu$ and $\nu$ will be denoted by $\mu * \nu$, and the adjoint of $\mu$ by $\mu^{*}$ i.e. if $\mu=\sum_{x \in G} \alpha(x) x, \mu^{*}=\sum_{x \in G} \bar{\alpha}(x) x^{-1}$. The first lemma is essentially Proposition 2.2 of [3].

LEMMA 2. If $\mu \in l_{1}(G)$ has norm-bounded convolution powers, then $\mu^{*}=\mu^{-1}$. In particular $\|\mu\|_{2}=1$.

We consider first the case where the identity $e$ of the group has the largest coefficient for all powers of $\mu$. (Here we use the phrase 'the largest coefficient' to mean a coefficient whose modulus is greater than or equal to the modulus of all other coefficients. Thus uniqueness is not implied and we avoid repetitive use of 'modulus'.)

We obtain a result which is slightly better than is required for this paper, but which is not best possible-see remark after Theorem 1 .

THEOREM 1. If $\mu \in l_{1}(G)$ has its powers norm-bounded by $5 / 3$, and if $e$ has the largest coefficient in $\mu^{2^{n}}$ for all $n$, then $\mu=\lambda$ e with $|\lambda|=1$.

Proof. Let $\mu^{2^{n}}=\alpha_{n} e+\beta_{n} u_{n}+f_{n}$ where $\left|\alpha_{n}\right| \geqslant\left|\beta_{n}\right| \geqslant$ all coefficients in $f_{n}$. Let $M=\sup _{n}\left|\beta_{n}\right|$, and fix $\varepsilon>0$. Choose $N$ such that $\left|\beta_{N}\right|>M /(1+\varepsilon)$. To simplify notation, we will write $\alpha$ for $\alpha_{N}, \beta$ for $\beta_{N}, u$ for $u_{N}, f$ for $f_{N}$. Then

$$
\begin{aligned}
\mu^{2^{N+1}} & =\mu^{2^{N}} * \mu^{2^{N}}=(\alpha e+\beta u+f) *(\alpha e+\beta u+f) \\
& =\alpha^{2} e+2 \alpha \beta u+\beta^{2} u^{2}+2 \alpha f+2 \beta u * f+f * f
\end{aligned}
$$


Now if $f=\Sigma \gamma(x) x$, the coefficient of $u$ in $f * f$ is $\Sigma_{x} \gamma(x u) \gamma\left(x^{-1}\right)$ and this has modulus $\leqslant \Sigma\left|\gamma(x u) \gamma\left(x^{-1}\right)\right| \leqslant \Sigma|\gamma(x)|^{2}=\|f\|_{2}^{2}$ by the Cauchy-Schwarz inequality. Hence the coefficient of $u$ in $\mu^{2^{N+1}}$ has modulus $>2|\alpha \beta|-\|f\|_{2}^{2}=2|\alpha \beta|-$ $\left(1-|\alpha|^{2}-|\beta|^{2}\right)=(|\alpha|+|\beta|)^{2}-1$, using the fact that $\left\|\mu^{2^{N}}\right\|_{2}=1$. By definition of $M$, we have

$$
(|\alpha|+|\beta|)^{2}-1 \leqslant M<|\beta|(1+\varepsilon) \text {, i.e. }|\alpha| \leqslant \vee(|\beta|+\varepsilon|\beta|+1)-|\beta| \text {. }
$$

But, by Lemma $1(\mathrm{~b}),|\beta| \geqslant\left(\left(1-|\alpha|^{2}\right) /(K-|\alpha|)\right)$ which decreases as $|\alpha|$ increases over the interval $[1 / K, K] .(|\alpha| \geqslant 1 / K$ by Lemma $1(\mathrm{a})$.) Hence

$$
|\beta| \geqslant \frac{1-[\vee(|\beta|+\varepsilon|\beta|+1)-|\beta|]^{2}}{K-[\vee(|\beta|+\varepsilon|\beta|+1)-|\beta|]}
$$

i.e.

$$
\begin{aligned}
& K|\beta|-|\beta| \vee(|\beta|+\varepsilon|\beta|+1)+|\beta|^{2} \\
& \geqslant 1-\left[|\beta|+\varepsilon|\beta|+1+|\beta|^{2}-2|\beta| \vee(|\beta|+\varepsilon|\beta|+1)\right]
\end{aligned}
$$

or

$$
2|\beta|^{2}+(K+1+\varepsilon)|\beta|-3|\beta| \vee(|\beta|+\varepsilon|\beta|+1) \geqslant 0 .
$$

Therefore $|\beta|=0$ or $2|\beta|+(K+1+\varepsilon)-3 \vee(|\beta|+|\beta| \varepsilon+1) \geqslant 0$. If $\varepsilon=0$, this last condition becomes

$$
(2|\beta|+K+1)^{2}>9(|\beta|+1)
$$

or

$$
\begin{aligned}
|\beta| & \geqslant \sqrt{ }\left(\frac{153}{64}-\frac{9 K}{8}\right)-\frac{1}{2}\left(K-\frac{5}{4}\right) \\
& \geqslant \frac{\sqrt{ } 33}{8}-\frac{5}{24} \quad \text { since } K<\frac{5}{3} \\
& >0.50974 .
\end{aligned}
$$

Thus, by continuity, there exists $\varepsilon>0$ for which the corresponding $\beta$ must satisfy either $|\beta|=0$ or $|\beta|>\frac{1}{2}$. In fact, since $|\alpha| \leqslant \sqrt{ }(|\beta|+\varepsilon|\beta|+1)-|\beta|$, we may assume that if $|\beta| \neq 0$, we have $|\alpha|<\sqrt{\frac{3}{2}}-\frac{1}{2}<0.725$. By Lemma 1 (c)

$$
|\alpha|+|\beta| \geqslant \frac{K+|\alpha|}{2}+\sqrt{ }\left[\frac{1}{2}\left(1-|\alpha|^{2}\right)-\frac{1}{4}(K-|\alpha|)^{2}\right]
$$

which, as in the proof of Lemma 1, decreases as $|\alpha|$ increases. Therefore

$$
|\alpha|+|\beta|>\frac{K+.725}{2}+\sqrt{ }\left[\frac{1}{2}\left(1-(.725)^{2}\right)-\frac{1}{4}(K-.725)^{2}\right] .
$$

This is a concave function of $K$, since the second derivative is negative, and so has its minimum value in $1 \leqslant K \leqslant \frac{5}{3}$ at $K=1$ or $K=\frac{5}{3}$. But at $K=1$, the value is $>1.329$ and at $K=\frac{5}{3}$, it is bigger than 1.32. Thus

$$
|\alpha|+|\beta|>1.32 \text {. }
$$

But this makes $M \geqslant(|\alpha|+|\beta|)^{2}-1>0.74$ which is a contradiction (since $\left|\alpha_{n}\right|^{2}$ $+\left|\beta_{n}\right|^{2} \leqslant 1$, and $\left|\alpha_{n}\right| \geqslant\left|\beta_{n}\right|$ must mean $\left|\beta_{n}\right| \leqslant 1 / \sqrt{ } 2$ ). This means that $|\beta|=0$, and hence $M=0$. The result is proved. 
This is clearly not the best possible result. The proof given here can be used for a number slightly bigger than $\frac{5}{3}$. However, it is conceivable that the result is true without any restriction on $K$.

CONJECTURE. If $\mu \in l_{1}(G)$ has norm-bounded powers and $e$ has the largest coefficient for all powers of $\mu$, then $\mu=\lambda e$ with $|\lambda|=1$.

We now look at the case when $e$ does not always have the largest coefficient. We show first that the only other elements of the group that appear with the largest coefficient in $\mu^{2^{n}}$, for some $n$, must have order 2 . We need two lemmas.

LEMMA 3. Let $\mu^{*}=\mu^{-1}$ and $\mu=\alpha e+\beta u+f$ with $u^{2} \neq e$ and $|\alpha| \geqslant|\beta| \geqslant$ all coefficients in $f$. Then

$$
|\alpha|+|\beta| \leqslant \frac{1}{2}(\|\mu\|+1) .
$$

Proof. Now $\mu^{*}=\bar{\alpha} e+\bar{\beta} u^{-1}+f^{*}$. By Lemma 2,

$$
|\alpha|^{2}+|\beta|^{2}+\|f\|_{2}^{2}=1
$$

Also $|\alpha|+|\beta|+\|f\|=\|\mu\|=K$ say. The coefficient of $u$ in $\mu * \mu^{*}$ is zero. We consider two cases.

(1) If $u^{3}=e$, let $f=\gamma u^{-1}+g$.

Then the coefficient of $u$ in $\mu * \mu^{*}$ gives

$$
|\bar{\alpha} \beta+\alpha \bar{\gamma}+\bar{\beta} \gamma| \leqslant\|g\|_{2}^{2} .
$$

Hence $|\alpha \beta| \leqslant(|\alpha|+|\beta|)|\gamma|+\left(\|f\|_{2}^{2}-|\gamma|^{2}\right)$, which is an increasing function of $|\gamma|$ for $0 \leqslant|\gamma| \leqslant\|f\|_{2}$. Hence $|\alpha \beta| \leqslant(|\alpha|+|\beta|)\|f\|_{2}$.

(2) If $u^{3} \neq e$ let $f=\gamma u^{-1}+\delta u^{2}+g$.

Then the coefficient of $u$ is $\mu * \mu^{*}$ gives

$$
|\bar{\alpha} \beta+\alpha \bar{\gamma}+\bar{\beta} \delta| \leqslant\left(\|f\|_{2}^{2}-|\gamma|^{2}\right)^{1 / 2}\left(\|f\|_{2}^{2}-|\delta|^{2}\right)^{1 / 2} \text {. }
$$

Hence $|\alpha \beta| \leqslant|\alpha||\gamma|+|\beta||\delta|+\left(\|f\|_{2}^{2}-|\gamma|^{2}\right)^{1 / 2}\left(\|f\|_{2}^{2}-|\delta|^{2}\right)^{1 / 2}$ which, for fixed $|\gamma|$, is an increasing function of $|\delta|$ for $0 \leqslant|\delta| \leqslant|\gamma|$. Hence, by symmetry

$$
|\alpha \beta| \leqslant(|\alpha|+|\beta|) \theta+\left(\|f\|_{2}^{2}-\theta^{2}\right), \quad \text { where } \theta=\max (|\gamma|,|\delta|) \text {. }
$$

Now, as in (1), since $\theta \leqslant\|f\|_{2},|\alpha \beta| \leqslant(|\alpha|+|\beta|)\|f\|_{2}$. Thus, in either case, we have $|\alpha \beta| \leqslant(|\alpha|+|\beta|)\|f\|_{2}$. Now

$$
|\alpha \beta|=\frac{1}{2}\left[(|\alpha|+|\beta|)^{2}-|\alpha|^{2}-|\beta|^{2}\right]=\frac{1}{2}\left[(|\alpha|+|\beta|)^{2}-1+\|f\|_{2}^{2}\right] .
$$

Therefore

$$
(|\alpha|+|\beta|)^{2}-1+\|f\|_{2}^{2} \leqslant 2(|\alpha|+|\beta|)\|f\|_{2}
$$

i.e.

$$
\left(|\alpha|+|\beta|-\|f\|_{2}\right)^{2}<1
$$

or

$$
|\alpha|+|\beta| \leqslant 1+\|f\|_{2} \leqslant 1+\|f\|_{1} \leqslant 1+(K-|\alpha|-|\beta|)
$$

and the result follows. 
LEMMA 4. If $\mu^{*}=\mu^{-1},\|\mu\|=K, \mu=\alpha e+\beta u+f$ where $|\alpha| \geqslant|\beta|>$ coefficients of $f$. Then $|\alpha|+|\beta| \leqslant(K+1) / 2$ implies that

$$
|\alpha|>\frac{1}{4}\{K+1+\sqrt{ }(K+1)(5-3 K)\} \text {. }
$$

Proof. By Lemma 1(c), either

$$
|\alpha|>\frac{K}{3}+\frac{2}{3} \sqrt{ }\left(\frac{3-K^{2}}{2}\right)
$$

or

$$
|\beta| \geqslant \frac{1}{2}(K-|\alpha|)+\vee\left\{\frac{1}{2}\left(1-|\alpha|^{2}\right)-\frac{1}{4}(K-|\alpha|)^{2}\right\}
$$

In the latter case,

$$
|\alpha|+|\beta| \geqslant \frac{1}{2}(K+|\alpha|)+\vee\left\{\frac{1}{2}\left(1-|\alpha|^{2}\right)-\frac{1}{4}(K-|\alpha|)^{2}\right\} .
$$

Since $|\alpha|+|\beta|<\frac{1}{2}(K+1)$, we have

$$
\frac{1}{2}(1-|\alpha|) \geqslant \vee\left\{\frac{1}{2}\left(1-|\alpha|^{2}\right)-\frac{1}{4}(K-|\alpha|)^{2}\right\} .
$$

Simplifying, we obtain

$$
\frac{1}{4}(K-|\alpha|)^{2}>\frac{1}{2}\left(1-|\alpha|^{2}\right)-\frac{1}{4}(1-|\alpha|)^{2}=\frac{1}{4}\left(1+2|\alpha|-3|\alpha|^{2}\right)
$$

or

$$
\frac{1}{4} K^{2} \geqslant \frac{1}{4}+\frac{1}{2}(K+1)|\alpha|-|\alpha|^{2}
$$

which becomes $|\alpha|>\frac{1}{4}\{K+1+\sqrt{ }(K+1)(5-3 K)\}$. Thus, the result is proved if we can show that

$$
\frac{1}{3} K+\frac{2}{3} \sqrt{ }\left(\frac{3-K^{2}}{2}\right)>\frac{1}{4}\{K+1+\sqrt{ }(K+1)(5-3 K)\}
$$

But this is equivalent to

$$
\begin{aligned}
\frac{4}{9}\left(\frac{3-K^{2}}{2}\right) & \geqslant\left\{\frac{3-K}{12}+\frac{1}{4} \sqrt{ }(K+1)(5-3 K)\right\}^{2} \\
& =\frac{3}{8}+\frac{1}{12} K-\frac{26}{144} K^{2}+\frac{1}{24}(3-K) \vee(K+1)(5-3 K)
\end{aligned}
$$

i.e.

$$
\left(7-2 K-K^{2}\right)>(3-K) \vee(K+1)(5-3 K)
$$

or

$$
\left(7-2 K-K^{2}\right)^{2} \geqslant(3-K)^{2}(K+1)(5-3 K) .
$$

But this is equivalent to $(K-1)^{4}>0$ which is certainly true.

From now on we assume that $K<\frac{1}{3}(4 \cos (\pi / 9)+1)$.

THEOREM 2. Let $\mu=\alpha e+\beta u+f, \mu^{*}=\mu^{-1}$, where $|\alpha|$ is the largest coefficient in $\mu$ and $u$ has the largest coefficient in $\mu^{2}$. If $\|\mu\| \leqslant K$ and $\left\|\mu^{2}\right\|<K$, then $|\beta|$ is the second largest coefficient in $\mu$ and $u^{2}=e$. 
Proof. Suppose that $|\beta|$ is not the second largest coefficient, and that $\mu=\alpha e+$ $\beta u+\gamma v+g$ where $|\gamma|$ is the second largest coefficient. We obtain a contradiction by considering two cases:

Case $1 .|\alpha| \leqslant 0.83$. By Lemma $1(\mathrm{c})$

$$
|\alpha|+|\gamma| \geqslant \frac{1}{2}(K+|\alpha|)+\vee\left\{\frac{1}{2}\left(1-|\alpha|^{2}\right)-\frac{1}{4}(K-|\alpha|)^{2}\right\}
$$

which, as in Lemma 1 , decreases as $|\alpha|$ increases. Therefore $|\alpha|+|\gamma| \geqslant 1$.32. But the coefficient of $v$ in $\mu^{2}$ has modulus

$$
\geqslant 2|\alpha \gamma|-\left(1-|\alpha|^{2}-|\gamma|^{2}\right)=(|\alpha|+|\gamma|)^{2}-1>0.74
$$

But this contradicts the fact that $u$ has the largest coefficient in $\mu^{2}$.

Case 2. $|\alpha|>0.83$. Now the coefficient of $e$ in $\mu^{2}$ has modulus $>|\alpha|^{2}-$ $\left(1-|\alpha|^{2}\right)=2|\alpha|^{2}-1>0.37$. By Lemma $1(\mathrm{f})$, this cannot be the third largest coefficient in $\mu^{2}$, so it must be the second. Thus the coefficient of $v$ in $\mu^{2}$ cannot be the second largest, and so by Lemma $1(f)$ again has modulus $\leqslant 0.36481$ i.e. $2|\alpha \gamma|-\left(1-|\alpha|^{2}-|\gamma|^{2}\right) \leqslant 0.36481$ or $|\alpha|+|\gamma| \leqslant \vee 1.365<1.169$. Now Lemma (b) implies that

$$
|\alpha|+|\gamma| \geqslant|\alpha|+\frac{1-|\alpha|^{2}}{K-|\alpha|}
$$

and so

$$
1+K|\alpha|-2|\alpha|^{2} \leqslant 1.169(K-|\alpha|)
$$

which implies $|\alpha|>0.9$ and $|\gamma|<0.269$. Since $|\beta|<|\gamma|,|\beta|<0.269$ and $|\alpha|-$ $|\beta|>0.63$. But the coefficient of $u$ in $\mu^{2}$ has modulus less than $2|\alpha \beta|+\left(1-|\alpha|^{2}\right.$ $\left.-|\beta|^{2}\right)=1-(|\alpha|-|\beta|)^{2}<0.61$. This again contradicts the fact that $u$ has the largest coefficient in $\mu^{2}$. Thus we have proved that $|\beta|$ is the second largest coefficient in $\mu$.

Now suppose that $u^{2} \neq e$. Then, by Lemma $3,|\alpha|+|\beta| \leqslant \frac{1}{2}(K+1)$. Also by Lemma 4,

$$
|\alpha|>\frac{1}{4}\{K+1+\vee(K+1)(5-3 K)\}
$$

which decreases with $K$, and so $|\alpha|>0.844$. Hence the coefficient of $e$ in $\mu^{2}$ has modulus greater than $2|\alpha|^{2}-1>0.424$. By Lemma $1(f)$, this must be the second largest coefficient in $\mu^{2}$. Thus we can apply Lemma 4 to $\mu^{2}$ to obtain that the coefficient of $u$ in $\mu^{2}$ has modulus greater than

$$
\frac{1}{4}(K+1)+\frac{1}{4} \vee(K+1)(5-3 K) .
$$

This modulus is less than $2|\alpha \beta|+\|f\|_{2}^{2}$. But

$$
\begin{aligned}
2|\alpha \beta|+\|f\|_{2}^{2} & =(|\alpha|+|\beta|)^{2}+2\|f\|_{2}^{2}-1 \\
& \leqslant(|\alpha|+|\beta|)^{2}+2(K-|\alpha|-|\beta|)^{2}-1
\end{aligned}
$$

which is a concave function of $|\alpha|+|\beta|$. Since $1 \leqslant|\alpha|+|\beta|<\frac{1}{2}(K+1)$ (see note after Lemma 1), we have

$$
2|\alpha \beta|+\|f\|_{2}^{2} \leqslant \max \left\{\left(\frac{K+1}{2}\right)^{2}+2\left(\frac{K-1}{2}\right)^{2}-1,2(K-1)^{2}\right\} .
$$


But

$$
\left(\frac{K+1}{2}\right)^{2}+2\left(\frac{K-1}{2}\right)^{2}-1=\frac{3}{4} K^{2}-\frac{1}{2} K-\frac{1}{4}>2(K-1)^{2}
$$

since $\left|K-\frac{7}{5}\right|^{2} \leqslant \frac{24}{25}$. Hence

$$
2|\alpha \beta|+\|f\|_{2}^{2} \leqslant \frac{3}{4} K^{2}-\frac{1}{2} K-\frac{1}{4}
$$

From (1) and (2)

$$
\frac{1}{4}(K+1)+\frac{1}{4} \sqrt{ }(K+1)(5-3 K) \leqslant \frac{3}{4} K^{2}-\frac{1}{2} K-\frac{1}{4}
$$

i.e.

$$
\frac{1}{16}(K+1)(5-3 K) \leqslant\left(\frac{3}{4} K^{2}-\frac{3}{4} K-\frac{1}{2}\right)^{2}
$$

or

$$
5+2 K-3 K^{2} \leqslant 9 K^{4}-18 K^{3}-3 K^{2}+12 K+4 .
$$

This is equivalent to

$$
9 K^{4}-18 K^{3}+10 K-1 \geqslant 0
$$

or

$$
(K-1)\left(9 K^{3}-9 K^{2}-9 K+1\right)>0 .
$$

But this is a contradiction, since $K<\frac{1}{3}(4 \cos (\pi / 9)+1)$ which is a solution of $\theta^{3}-\theta^{2}-\theta+\frac{1}{9}=0$. This contradiction proves Theorem 2 .

REMARK. This proof is an improvement on Lemma 2.4 of [3]. In fact part of the proof there of the fact that $u^{2}=e$ is incorrect, but can easily be rectified. In estimating the coefficient of $u^{-1}$, it should be

$$
|\alpha \bar{\beta}| \leqslant(|\alpha|+|\beta|)\|f\|+\|f\|^{2}
$$

which implies that $|\beta|<0.1947$. But this still contradicts $|\beta|>0.37$.

It remains to show that it is always the same element of order 2 that appears. We use the idea of Lemma 2.5 in [3], but the estimates have to be made very carefully. In fact, in the general case, that method only shows that at most a finite number of elements of order 2 appear. This turns out to be sufficient if we can characterize all measures with bounded powers supported on the Klein 4-group. We shall do this first.

Let $G=\{e, u, v, u v\}$ with $u^{2}=v^{2}=(u v)^{2}=e$. Then the measures on $G$ with powers norm-bounded have the form

$$
\mu=\lambda_{0} \chi_{0}+\lambda_{1} \chi_{1}+\lambda_{2} \chi_{2}+\lambda_{3} \chi_{3},
$$

where $\chi_{0}, \chi_{1}, \chi_{2}$ and $\chi_{3}$ are the characters on $G$ and $\left|\lambda_{0}\right|=\left|\lambda_{1}\right|=\left|\lambda_{2}\right|=\left|\lambda_{3}\right|=1$. i.e.

$$
\begin{aligned}
\mu= & \frac{1}{4}\left(\lambda_{0}+\lambda_{1}+\lambda_{2}+\lambda_{3}\right) e+\frac{1}{4}\left(\lambda_{0}-\lambda_{1}+\lambda_{2}-\lambda_{3}\right) u \\
& +\frac{1}{4}\left(\lambda_{0}+\lambda_{1}-\lambda_{2}-\lambda_{3}\right) v+\frac{1}{4}\left(\lambda_{0}-\lambda_{1}-\lambda_{2}+\lambda_{3}\right) u v .
\end{aligned}
$$

Now if $K=\sup _{n}\left\|\mu^{n}\right\|$, we have an explicit formula for $K$ :

$$
\begin{aligned}
K=\sup _{n}\left\{\frac{1}{4} \mid \lambda_{0}^{n}+\lambda_{1}^{n}+\right. & \lambda_{2}^{n}+\lambda_{3}^{n}\left|+\frac{1}{4}\right| \lambda_{0}^{n}-\lambda_{1}^{n}+\lambda_{2}^{n}-\lambda_{3}^{n} \mid \\
& \left.+\frac{1}{4}\left|\lambda_{0}^{n}+\lambda_{1}^{n}-\lambda_{2}^{n}-\lambda_{3}^{n}\right|+\frac{1}{4}\left|\lambda_{0}^{n}-\lambda_{1}^{n}-\lambda_{2}^{n}+\lambda_{3}^{n}\right|\right\} .
\end{aligned}
$$


However this is not a very useful formula. We will show that if

$$
K<\frac{1}{3}(4 \cos (\pi / 9)+1)
$$

then $\mu$ is supported on a subgroup of order 2 . It is easy to check that $\mu$ is supported on a subgroup of order 2 if the $\lambda_{i}$ 's are either equal in pairs or add up to zero in pairs. It seems likely that the best result here is that if $\mu$ is not supported on a subgroup of order 2 , then $K \geqslant \frac{1}{4}+\sin (2 \pi / 5)+\sin (2 \pi / 5) \sin (\pi / 5) \sim 1.76$, and this value is attained when $\lambda_{0}=1, \lambda_{1}=\exp (2 \pi i / 5), \lambda_{2}=\exp (4 \pi i / 5), \lambda_{3}=\exp (6 \pi i / 5)$, but the weaker result is enough here.

It is clear that if two of the $\lambda_{i}$ 's are equal then $\mu$ has two coefficients adding up to zero. We show now that this only occurs if $K>1+\frac{1}{2} \sqrt{ } 3 \sim 1.86$. This value is attained when, for example, $\lambda_{0}=\lambda_{1}=1, \lambda_{2}=\exp (2 \pi i / 3), \lambda_{3}=\exp (4 \pi i / 3)$. The significance of this example is that only one element of order 2 appears as the largest coefficient in the powers of $\mu$, but it is not supported on a group of order 2 .

LEMMA 5. If $\mu=\alpha e+\beta u+\gamma v-\gamma u v, \gamma \neq 0$ and $K=\sup _{n}\left\|\mu^{n}\right\|$, then $K \geqslant 1+\frac{1}{2} \sqrt{ } 3$.

Proof. We have $|\alpha|^{2}+|\beta|^{2}+2|\gamma|^{2}=1$ by Lemma 2 , and $|\alpha+\beta|=1$ by applying the identity character to $\mu$. Also if $\mu^{2}=\alpha_{1} e+\beta_{1} u+\gamma_{1} v-\gamma_{1} u v$, we have $\gamma_{1}=2 \gamma(\alpha-\beta)$. Now $|\alpha-\beta|^{2}=2|\alpha|^{2}+2|\beta|^{2}-|\alpha+\beta|^{2}=2\left(1-2|\gamma|^{2}\right)-$ $1=1-4|\gamma|^{2}$. Suppose $K<1+\frac{1}{2} \sqrt{ } 3$. Then $2|\gamma|<K-|\alpha|-|\beta|<K-1$ i.e. $4|\gamma|^{2}<(K-1)^{2}$ and so $|\alpha-\beta|^{2}>1-(K-1)^{2}$. Therefore $\left|\gamma_{1}\right|=2|\gamma(\alpha-\beta)|>$ $|\gamma|\left\{2 \sqrt{ }\left(1-(K-1)^{2}\right)\right\}$. If $2 \sqrt{ }\left(1-(K-1)^{2}\right)=M, M>1$ since $K<1+\frac{1}{2} \sqrt{ } 3$. Hence if $\mu^{n+1}=\alpha_{n} e+\beta_{n} u+\gamma_{n} v-\gamma_{n} u v$ we have $\left|\gamma_{n}\right|>M^{n}|\gamma|$ which is a contradiction. Hence $\gamma=0$. This completes the proof.

We need the following lemmas:

LEMMA 6. If $\mu=\alpha e+\beta u+\gamma v+\delta u v$ and $\mu^{-1}=\mu^{*}$, then, if $|\alpha| \geqslant|\beta|>|\gamma|>$ $|\delta|$,

(i) $|\alpha \delta| \geqslant|\beta \gamma|$;

(ii) $|\alpha \beta+\gamma \delta| \leqslant \sqrt{ }\left(|\alpha \beta|^{2}-|\gamma \delta|^{2}\right) \leqslant|\alpha \beta|$;

(iii) If $|\alpha|+|\beta| \leqslant A$, then

$$
|\alpha| \geqslant \frac{A}{2}+\sqrt{ }\left\{\frac{1}{2}\left[\left(\frac{K}{A}-1\right)^{2}+1\right]^{-1}-\frac{1}{4} A^{2}\right\}
$$

(iv)

$$
|\alpha|+|\beta| \leqslant K\left(1+\sqrt{\left.\left(\frac{|\gamma|^{2}+|\delta|^{2}}{1-|\gamma|^{2}-|\delta|^{2}}\right)\right)^{-1}}\right.
$$

(v)

$$
\left|\frac{\alpha \bar{\beta}}{|\alpha|}+\frac{\bar{\alpha} \beta}{|\beta|}\right| \leqslant \vee\left\{2-(|\alpha|+|\beta|)^{2}\right\} .
$$

Further, if $\mu^{2}=\alpha_{1} e+\beta_{1} u+\gamma_{1} v+\delta_{1} u v$, then

(vi) $\left|\gamma_{1}\right|^{2}+\left|\delta_{1}\right|^{2} \geqslant 4\left(|\gamma|^{2}+|\delta|^{2}\right)\left(1-2|\gamma|^{2}-2|\delta|^{2}\right)$. 
Proof. Without loss of generality, we may assume that $\alpha$ is real. Then the condition $\mu^{*}=\mu^{-1}$ gives

$$
\begin{aligned}
& \alpha(\beta+\bar{\beta})+\gamma \bar{\delta}+\bar{\gamma} \delta=0, \\
& \alpha(\gamma+\bar{\gamma})+\beta \bar{\delta}+\bar{\beta} \delta=0, \\
& \alpha(\delta+\bar{\delta})+\beta \bar{\gamma}+\bar{\beta} \gamma=0 .
\end{aligned}
$$

If $\delta=0$, then $\beta+\bar{\beta}=\gamma+\bar{\gamma}=\beta \bar{\gamma}+\bar{\beta} \gamma=0$, which implies $\gamma=0$. Thus (i) and (ii) hold trivially.

Put $\beta=|\beta| e^{i \psi}, \gamma=|\gamma| e^{i \theta}$ and $\delta=|\delta| e^{i \phi}$. Then

$$
|\alpha \beta| \cos \psi+|\gamma \delta| \cos (\theta-\phi)=0 \text {. }
$$

Hence

$$
(|\alpha \beta|-|\gamma \delta|) \cos \psi+|\gamma \delta|(\cos \psi+\cos (\theta-\phi))=0
$$

or

$$
(|\alpha \beta|-|\gamma \delta|) \cos \psi+2|\gamma \delta| \cos \frac{1}{2}(\psi-\theta+\phi) \cos \frac{1}{2}(\psi+\theta-\phi)=0 .
$$

Similarly

$$
(|\alpha \beta|+|\gamma \delta|) \cos \psi+2|\gamma \delta| \sin \frac{1}{2}(\psi-\theta+\phi) \sin \frac{1}{2}(\psi+\theta-\phi)=0 .
$$

Combining these, we get, either $\cos \psi=0$ or

$$
\frac{|\alpha \beta|+|\gamma \delta|}{|\alpha \beta|-|\gamma \delta|}=\tan \frac{1}{2}(\psi-\theta+\phi) \tan \frac{1}{2}(\psi+\theta-\phi) .
$$

If $\cos \psi=0, \cos (\theta-\phi)=0$ and so $\psi= \pm \pi / 2, \theta-\phi= \pm \pi / 2$. Substituting in (2) and (3) we get

$$
|\alpha \gamma| \cos \theta \pm|\beta \delta| \sin \phi=0 \text { and }|\alpha \delta| \cos \phi \pm|\beta \gamma| \sin \theta=0 .
$$

Since $\cos \theta= \pm \sin \phi$, this gives

$$
(|\alpha \gamma| \pm|\beta \delta|) \sin \phi=0 \text { and }(|\alpha \delta| \pm|\beta \gamma|) \sin \theta=0 .
$$

Therefore either $|\alpha \gamma|=|\beta \delta|$ or $|\alpha \delta|=|\beta \gamma|$. But since $|\alpha|>|\beta|>|\gamma|>|\delta|$, both these imply $|\alpha \delta|=|\beta \gamma|$.

From (2) we obtain that either $\cos \theta=0$ or

$$
\frac{|\alpha \gamma|+|\beta \delta|}{|\alpha \gamma|-|\beta \delta|}=\tan \frac{1}{2}(\theta-\phi+\psi) \tan \frac{1}{2}(\theta+\phi-\psi) \text {. }
$$

If $\cos \theta=0$, a similar argument to above shows that $|\alpha \delta|=|\beta \gamma|$. From (3), $\cos \phi=0$ or

$$
\frac{|\alpha \delta|+|\beta \gamma|}{|\alpha \delta|-|\beta \gamma|}=\tan \frac{1}{2}(\phi-\psi+\theta) \tan \frac{1}{2}(\phi+\psi-\theta) .
$$

Again if $\cos \phi=0$, we obtain $|\alpha \delta|=|\beta \gamma|$. In the other case, by multiplication,

$$
\begin{aligned}
& \frac{(|\alpha \beta|+|\gamma \delta|)(|\alpha \gamma|+|\beta \delta|)(|\alpha \delta|+|\beta \gamma|)}{(|\alpha \beta|-|\gamma \delta|)(|\alpha \gamma|-|\beta \delta|)(|\alpha \delta|-|\beta \gamma|)} \\
& \quad=\tan ^{2} \frac{1}{2}(\psi-\theta+\phi) \tan ^{2} \frac{1}{2}(\theta-\psi+\phi) \tan ^{2} \frac{1}{2}(\theta-\phi+\psi)>0 .
\end{aligned}
$$


In particular $|\alpha \delta| \geqslant|\beta \gamma|$.

(ii)

$$
\begin{aligned}
|\alpha \beta+\gamma \delta|^{2}= & |\alpha \beta|^{2}+\alpha \beta \bar{\gamma} \bar{\delta}+\alpha \bar{\beta} \gamma \delta+|\gamma \delta|^{2}=|\alpha \beta|^{2} \\
& +|\gamma \delta|^{2}+2|\alpha \beta \gamma \delta| \cos (\psi-\theta-\phi) .
\end{aligned}
$$

But

$$
\cos (\psi-\theta-\phi)=\frac{1-\tan ^{2} \frac{1}{2}(\psi-\theta-\phi)}{1+\tan ^{2} \frac{1}{2}(\psi-\theta-\phi)}
$$

and

$$
\tan ^{2} \frac{1}{2}(\psi-\theta-\phi)=\frac{(|\alpha \gamma|+|\beta \delta|)(|\alpha \delta|+|\beta \gamma|)(|\alpha \beta|-|\gamma \delta|)}{(|\alpha \gamma|-|\beta \delta|)(|\alpha \delta|-|\beta \gamma|)(|\alpha \beta|+|\gamma \delta|)} .
$$

(If any of $\cos \theta, \cos \psi$ or $\cos \phi$ is zero, $|\alpha \delta|=|\beta \gamma|$, and this quantity is $\infty$.) Therefore

$$
\cos (\psi-\theta-\phi)=\frac{|\alpha \beta \gamma \delta|\left(1 /|\alpha|^{2}+1 /|\beta|^{2}-1 /|\gamma|^{2}-1 /|\delta|^{2}\right)}{|\alpha|^{2}+|\beta|^{2}-|\gamma|^{2}-|\delta|^{2}}<0 .
$$

In order to show that $|\alpha \beta+\gamma \delta|<|\alpha \beta|$, we need that

$$
|\gamma \delta|^{2} \leqslant-2|\alpha \beta \gamma \delta|^{2}\left(\frac{1 /|\alpha|^{2}+1 /|\beta|^{2}-1 /|\gamma|^{2}-1 /|\delta|^{2}}{|\alpha|^{2}+|\beta|^{2}-|\gamma|^{2}-|\delta|^{2}}\right) .
$$

But this is equivalent to

$$
|\alpha|^{2}+|\beta|^{2}-|\gamma|^{2}-|\delta|^{2} \leqslant-2|\beta|^{2}-2|\alpha|^{2}+2\left|\alpha^{2} \beta^{2}\right| \frac{\left(|\gamma|^{2}+|\delta|^{2}\right)}{|\gamma \delta|^{2}}
$$

i.e.

or

$$
2|\alpha|^{2}+2|\beta|^{2}-1 \leqslant-2|\beta|^{2}-2|\alpha|^{2}+2\left|\alpha^{2} \beta^{2}\right| \frac{\left(|\gamma|^{2}+|\delta|^{2}\right)}{|\gamma \delta|^{2}}
$$

$$
4|\alpha|^{2}+4|\beta|^{2}-1 \leqslant \frac{2\left|\alpha^{2} \beta^{2}\right|}{|\gamma \delta|^{2}}\left(|\gamma|^{2}+|\delta|^{2}\right) .
$$

But $|\gamma|^{2}+|\delta|^{2} \geqslant 2|\gamma \delta|$. Therefore

$$
\frac{|\gamma|^{2}+|\delta|^{2}}{|\gamma \delta|^{2}} \geqslant \frac{4}{|\gamma|^{2}+|\delta|^{2}}=\frac{4}{1-|\alpha|^{2}-|\beta|^{2}} .
$$

Hence

$$
2\left|\alpha^{2} \beta^{2}\right| \frac{\left(|\gamma|^{2}+|\delta|^{2}\right)}{|\gamma \delta|^{2}} \geqslant \frac{8\left|\alpha^{2} \beta^{2}\right|}{1-|\alpha|^{2}-|\beta|^{2}} .
$$

Thus it suffices to show that

$$
8|\alpha \beta|^{2} \geqslant\left(1-|\alpha|^{2}-|\beta|^{2}\right)\left(4|\alpha|^{2}+4|\beta|^{2}-1\right)
$$

i.e.

$$
4\left(|\alpha|^{2}+|\beta|^{2}\right)^{2}+8|\alpha \beta|^{2}-5\left(|\alpha|^{2}+|\beta|^{2}\right)+1>0
$$


i.e.

$$
\left(4|\alpha|^{2}+|\beta|^{2}-1\right)\left(|\alpha|^{2}+4|\beta|^{2}-1\right)>|\alpha \beta|^{2} .
$$

But since $|\alpha|$ and $|\beta|$ are the largest coefficients

$$
4|\alpha|^{2}+|\beta|^{2}-1 \geqslant|\alpha|^{2} \text { and }|\alpha|^{2}+4|\beta|^{2}-1 \geqslant|\beta|^{2} \text {. }
$$

This completes the proof.

(iii) We have

$$
\begin{gathered}
|\alpha|^{2}+|\beta|^{2}+|\gamma|^{2}+|\delta|^{2}=1 \\
|\alpha \delta| \geqslant|\beta \gamma| \\
|\alpha|+|\beta|+|\gamma|+|\delta|<K .
\end{gathered}
$$

Eliminating $|\delta|$ from (4) and (5) gives an upper bound for $|\gamma|^{2}$, namely

$$
\frac{|\alpha|^{2}\left(1-|\alpha|^{2}-|\beta|^{2}\right)}{|\alpha|^{2}+|\beta|^{2}}
$$

Eliminating $|\delta|$ from (4) and (6) gives a lower bound for $|\gamma|$, namely

$$
\frac{K-(|\alpha|+|\beta|)}{2}+\sqrt{ }\left\{\frac{1-|\alpha|^{2}-|\beta|^{2}}{2}-\left(\frac{K-(|\alpha|+|\beta|)}{2}\right)^{2}\right\}
$$

Combining these, we obtain the inequality

$$
\left(|\alpha|^{2}+|\beta|^{2}\right)\left[1+\left(\frac{K}{|\alpha|+|\beta|}-1\right)^{2}\right]>1 \text {. }
$$

Putting $|\alpha|+|\beta|=A$ and solving for $|\alpha|$, we get

$$
|\alpha| \geqslant \frac{A}{2}+\sqrt{ }\left\{\frac{1}{2}\left[\left(\frac{K}{A}-1\right)^{2}+1\right]^{-1}-\frac{A^{2}}{4}\right\}
$$

Since this is a decreasing function of $A$, the result follows.

(iv) is simply the inequality (7) above rewritten in terms of $|\alpha|+|\beta|$ and using $|\alpha|^{2}+|\beta|^{2}=1-|\gamma|^{2}-|\delta|^{2}$.

(v) $\left.|\alpha \bar{\beta} /| \alpha|+\bar{\alpha} \beta /| \beta\right|^{2}=|\alpha|^{2}+|\beta|^{2}+(1 /|\alpha \beta|)\left(\alpha^{2} \bar{\beta}^{2}+\bar{\alpha}^{2} \beta^{2}\right)$. But $|\alpha \bar{\beta}+\bar{\alpha} \beta|$ $\leqslant 2|\gamma \delta|$ by (1). Therefore $|\alpha \bar{\beta}+\bar{\alpha} \beta|^{2}=2|\alpha \beta|^{2}+\alpha^{2} \bar{\beta}^{2}+\bar{\alpha}^{2} \beta^{2}<4|\gamma \delta|^{2}$. Thus

$$
\begin{aligned}
\left|\frac{\alpha \bar{\beta}}{|\alpha|}+\frac{\bar{\alpha} \beta}{|\beta|}\right|^{2} & \leqslant|\alpha|^{2}+|\beta|^{2}+\frac{1}{|\alpha \beta|}\left(4|\gamma \delta|^{2}-2|\alpha \beta|^{2}\right) \\
& =(|\alpha|+|\beta|)^{2}+\frac{4}{|\alpha \beta|}\left(|\gamma \delta|^{2}-|\alpha \beta|^{2}\right) \\
& =(|\alpha|+|\beta|)^{2}+\frac{4|\gamma \delta|}{|\alpha \beta|}(|\gamma \delta|-|\alpha \beta|)+4(|\gamma \delta|-|\alpha \beta|) \\
& \leqslant(|\alpha|+|\beta|)^{2}-4(|\alpha \beta|-|\gamma \delta|) .
\end{aligned}
$$

Now $2|\gamma \delta| \leqslant|\gamma|^{2}+|\delta|^{2}=1-|\alpha|^{2}-|\beta|^{2}$. Therefore

$$
2|\gamma \delta|-2|\alpha \beta|<1-(|\alpha|+|\beta|)^{2}
$$


i.e.

$$
\left|\frac{\alpha \bar{\beta}}{|\alpha|}+\frac{\bar{\alpha} \beta}{|\beta|}\right|^{2} \leqslant(|\alpha|+|\beta|)^{2}+2\left[1-(|\alpha|+|\beta|)^{2}\right]=2-(|\alpha|+|\beta|)^{2}
$$

as required.

(vi) $\gamma_{1}=2 \alpha \gamma+2 \beta \delta$ and $\delta_{1}=2 \alpha \delta+2 \beta \gamma$. Therefore

$$
\begin{aligned}
\left|\gamma_{1}\right|^{2}+\left|\delta_{1}\right|^{2} & =4|\alpha \gamma+\beta \delta|^{2}+4|\alpha \delta+\beta \gamma|^{2} \\
& =4\left\{|\alpha \gamma|^{2}+|\beta \delta|^{2}+|\alpha \delta|^{2}+|\beta \gamma|^{2}+\alpha \gamma \bar{\beta} \bar{\delta}+\bar{\alpha} \bar{\gamma} \beta \delta+\alpha \delta \bar{\beta} \bar{\gamma}+\bar{\alpha} \bar{\delta} \beta \gamma\right\} \\
& =4\left\{\left(|\alpha|^{2}+|\beta|^{2}\right)\left(|\gamma|^{2}+|\delta|^{2}\right)+(\alpha \bar{\beta}+\bar{\alpha} \beta)(\gamma \bar{\delta}+\bar{\gamma} \delta)\right\} \\
& \geqslant 4\left\{\left(|\alpha|^{2}+|\beta|^{2}\right)\left(|\gamma|^{2}+|\delta|^{2}\right)-4|\gamma \delta|^{2}\right\} \quad \text { by (1). }
\end{aligned}
$$

But $2|\gamma \delta| \leqslant|\gamma|^{2}+|\delta|^{2}$, and so

$$
\begin{aligned}
\left|\gamma_{1}\right|^{2}+\left|\delta_{1}\right|^{2} & \geqslant 4\left\{\left(|\gamma|^{2}+|\delta|^{2}\right)\left(|\alpha|^{2}+|\beta|^{2}-|\gamma|^{2}-|\delta|^{2}\right)\right\} \\
& =4\left(|\gamma|^{2}+|\delta|^{2}\right)\left(1-2|\gamma|^{2}-2|\delta|^{2}\right)
\end{aligned}
$$

as required.

Lemma 7. If $\mu=\alpha e+\beta u+\gamma v+\delta u v, \mu^{*}=\mu^{-1},\|\mu\|<K$ and $u$ has the largest coefficient in $\mu^{2}$. Then if $|\alpha|+|\beta|<1.355,|\alpha| \geqslant 0.855$.

Proof. Suppose that $|\alpha|+|\beta|<1.355$ and that $\alpha<0.855$. Then $|\alpha|^{2}+|\beta|^{2}<$ $(0.855)^{2}+(0.5)^{2}<0.98101$. Hence $|\gamma|^{2}+|\delta|^{2}>0.0189$. By Lemma 6(vi), if $\mu^{2}=$ $\alpha_{1} u+\beta_{1} e+\gamma_{1} v+\delta_{1} u v$,

$$
\left|\gamma_{1}\right|^{2}+\left|\delta_{1}\right|^{2} \geqslant 4\left\{\left(|\gamma|^{2}+|\delta|^{2}\right)\left(1-2|\gamma|^{2}-2|\delta|^{2}\right)\right\}>0.0727 .
$$

By Lemma 6(iv) $\left|\alpha_{1}\right|+\left|\beta_{1}\right| \leqslant 1.240$. By Lemma 6(iii) $\left|\alpha_{1}\right|>0$.9. But $\alpha_{1}$ is the coefficient of $u$, i.e. $\alpha_{1}=2 \alpha \beta+2 \gamma \delta$. By Lemma 6(ii), $\left|\alpha_{1}\right|=2|\alpha \beta+\gamma \delta|<2|\alpha \beta|$. Now since $|\alpha|+|\beta|<1.355$, by Lemma 6 (iii) $|\alpha|>0.84$ and so $2|\alpha \beta|<0.866$. This makes $\left|\alpha_{1}\right|<0.866$ which is a contradiction.

LEMMA 8. Let $\mu=\alpha e+\beta u+\gamma v+\delta u v$ and $\mu^{2}=\alpha_{1} u+\beta_{1} e+\gamma_{1} v+\delta_{1} u v$, where $|\alpha|$ and $\left|\alpha_{1}\right|$ are the largest coefficients in $\mu$ and $\mu^{2}$ respectively. If $\mu^{*}=\mu^{-1}$ and $\|\mu\| \leqslant K,\left\|\mu^{2}\right\|<K$, then either $|\alpha|+|\beta|>1.355$ or $\left|\alpha_{1}\right|+\left|\beta_{1}\right|>1.355$.

Proof. Suppose that $|\alpha|+|\beta|<1.355$. By Lemma 7, $|\alpha| \geqslant 0.855$ and $|\beta|<0.5$. Therefore

$$
\left|\alpha_{1}\right|=2|\alpha \beta+\gamma \delta|<2|\alpha \beta|<0.855 \text {. }
$$

It follows from Lemma 7 , that $\left|\alpha_{1}\right|+\left|\beta_{1}\right| \geqslant 1.355$ provided we can show that $u$ has the largest coefficient in $\mu^{4}$. Suppose that, on the contrary, $\left|\alpha_{1}\right|+\left|\beta_{1}\right|<1.355$ and that $u$ does not have the largest coefficient in $\mu^{4}$. Now $\left|\beta_{1}\right|>2|\alpha|^{2}-1>0.46$ and so is the second largest coefficient in $\mu^{2}$. Hence, by Lemma $1(\mathrm{c})$

$$
\left|\alpha_{1}\right|+\left|\beta_{1}\right| \geqslant \frac{1}{2}\left(K+\left|\beta_{1}\right|\right)+\vee\left\{\frac{1}{2}\left(1-\left|\beta_{1}\right|^{2}\right)-\frac{1}{4}\left(K-\left|\beta_{1}\right|\right)^{2}\right\}>1.300 .
$$

Therefore if $\mu^{4}=\alpha_{2} e+\beta_{2} u+\gamma_{2} v+\delta_{2} u v, \beta_{2}=2\left(\alpha_{1} \beta_{1}+\gamma_{1} \delta_{1}\right)$ and so

$$
\begin{aligned}
\left|\beta_{2}\right| & \geqslant 2\left|\alpha_{1} \beta_{1}\right|-2\left|\gamma_{1} \delta_{1}\right| \geqslant 2\left|\alpha_{1} \beta_{1}\right|-\left(\left|\gamma_{1}\right|^{2}+\left|\delta_{1}\right|^{2}\right) \\
& =\left(\left|\alpha_{1}\right|+\left|\beta_{1}\right|\right)^{2}-1>0.69 .
\end{aligned}
$$


However, as in the proof of Lemma 7, applied to $\mu^{2}$ in place of $\mu$, since $\left|\alpha_{1}\right|+\left|\beta_{1}\right| \leqslant 1.355$ and $\left|\alpha_{1}\right|<0.855$ we have $\left|\alpha_{2}\right|+\left|\beta_{2}\right|<1.24$ and $\left|\alpha_{2}\right|>0.9$. Hence $\left|\beta_{2}\right|<0.34$ which is a contradiction.

THEOREM 3. If $\mu \in l_{1}\left(\mathbf{Z}_{2} \times \mathbf{Z}_{2}\right)$ has powers norm bounded by

$$
K<\frac{1}{3}(4 \cos (\pi / 9)+1)
$$

then $\mu$ is supported on a subgroup of order 2 .

Proof. We show first that only one element of order 2 can appear the largest coefficient in $\mu^{ \pm 2^{n}}$. Suppose $u$ and $v$ both appear. By Lemma 10, there exist powers of $\mu$ such that

$$
\mu^{n}=\alpha_{1} e+\beta_{1} u+\gamma_{1} v+\delta_{1} u v \text { and } \mu^{m}=\alpha_{2} e+\beta_{2} v+\gamma_{2} u+\delta_{2} u v
$$

where $\left|\alpha_{1}\right|+\left|\beta_{1}\right| \geqslant 1.355$ and $\left|\alpha_{2}\right|+\left|\beta_{2}\right| \geqslant 1.355$. (Here, we only assume that $\alpha_{1}$ and $\beta_{1}$ are the two largest coefficients.) As in [3, Lemma 2.5], we estimate $\left\|\mu^{m+n}\right\|$.

$$
\begin{aligned}
\left\|\mu^{m+n}\right\| & =\left|\alpha_{1} \alpha_{2}+\beta_{1} \gamma_{2}+\beta_{2} \gamma_{1}+\delta_{1} \delta_{2}\right|+\left|\alpha_{1} \beta_{2}+\alpha_{2} \gamma_{1}+\beta_{1} \delta_{2}+\gamma_{2} \delta_{1}\right| \\
& +\left|\alpha_{2} \beta_{1}+\alpha_{1} \gamma_{2}+\gamma_{1} \delta_{2}+\beta_{2} \delta_{1}\right|+\left|\beta_{1} \beta_{2}+\alpha_{1} \delta_{2}+\alpha_{2} \delta_{1}+\gamma_{1} \gamma_{2}\right| \\
\geqslant & \left|\alpha_{1} \alpha_{2}\right|\left(1+\operatorname{Re} \frac{\beta_{1} \gamma_{2}+\beta_{2} \gamma_{1}+\delta_{1} \delta_{2}}{\alpha_{1} \alpha_{2}}\right)+\left|\alpha_{1} \beta_{2}\right|\left(1+\operatorname{Re} \frac{\alpha_{2} \gamma_{1}+\beta_{1} \delta_{2}+\gamma_{2} \delta_{1}}{\alpha_{1} \beta_{2}}\right) \\
& +\left|\alpha_{2} \beta_{1}\right|\left(1+\operatorname{Re} \frac{\alpha_{1} \gamma_{2}+\gamma_{1} \delta_{2}+\beta_{2} \delta_{1}}{\alpha_{2} \beta_{1}}\right) \\
& +\left|\beta_{1} \beta_{2}\right|\left(1+\operatorname{Re} \frac{\alpha_{1} \delta_{2}+\alpha_{2} \delta_{1}+\gamma_{1} \gamma_{2}}{\beta_{1} \beta_{2}}\right) \\
\geqslant & \left(\left|\alpha_{1}\right|+\left|\beta_{1}\right|\right)\left(\left|\alpha_{2}\right|+\left|\beta_{2}\right|\right)+\operatorname{Re}\left\{\gamma_{1}\left(\frac{\left|\alpha_{1} \alpha_{2}\right|}{\alpha_{1} \alpha_{2}} \beta_{2}+\frac{\left|\alpha_{1} \beta_{2}\right|}{\alpha_{1} \beta_{2}} \alpha_{2}\right)\right\} \\
& +\operatorname{Re}\left\{\gamma_{2}\left(\frac{\left|\alpha_{1} \alpha_{2}\right|}{\alpha_{1} \alpha_{2}} \beta_{1}+\frac{\mid \alpha_{2} \beta_{1}}{\beta_{2} \beta_{1}} \alpha_{1}\right)\right\}+\operatorname{Re}\left\{\delta_{1}\left(\frac{\left|\alpha_{2} \beta_{1}\right|}{\alpha_{2} \beta_{1}} \beta_{2}+\frac{\left|\beta_{1} \beta_{2}\right|}{\beta_{1} \beta_{2}} \alpha_{2}\right)\right\} \\
& +\operatorname{Re}\left\{\delta_{2}\left(\frac{\left|\alpha_{1} \beta_{2}\right|}{\alpha_{1} \beta_{2}} \beta_{1}+\frac{\left|\beta_{1} \beta_{2}\right|}{\beta_{1} \beta_{2}} \alpha_{1}\right)\right\}-\left|\delta_{1} \delta_{2}\right|-\left|\gamma_{2} \delta_{1}\right|-\left|\gamma_{1} \delta_{2}\right|-\left|\gamma_{1} \gamma_{2}\right| \\
\geqslant & \left(\left|\alpha_{1}\right|+\left|\beta_{1}\right|\right)\left(\left|\alpha_{2}\right|+\left|\beta_{2}\right|\right)-\left|\gamma_{1}\right|\left|\frac{\alpha_{2} \beta_{2}}{\left|\alpha_{2}\right|}+\frac{\alpha_{2} \bar{\beta}_{2}}{\left|\beta_{2}\right|}\right| \\
& \left.-\left|\gamma_{2}\right|\left|\frac{\alpha_{1} \beta_{1}}{\left|\alpha_{1}\right|}+\frac{\alpha_{1} \bar{\beta}_{1}}{\left|\beta_{1}\right|}\right|-\left|\delta_{1}\right|\left|\frac{\alpha_{2} \beta_{2}}{\left|\alpha_{2}\right|}+\frac{\alpha_{2} \bar{\beta}_{2} \mid}{\left|\beta_{2}\right|}\right|-\left|\delta_{2}\right|\left|\frac{\alpha_{1} \beta_{1}}{|\alpha|}+\frac{\alpha_{1} \bar{\beta}_{1} \mid}{|\beta|}\right|+\left|\delta_{1}\right|\right)\left(\left|\gamma_{2}\right|+\left|\delta_{2}\right|\right) \\
& -\left(\left|\gamma_{1}\right|\right.
\end{aligned}
$$

which, by Lemma $6(v)$ is greater than

$$
\begin{aligned}
\left(\left|\alpha_{1}\right|+\left|\beta_{1}\right|\right)\left(\left|\alpha_{2}\right|+\left|\beta_{2}\right|\right)-\left(\left|\gamma_{1}\right|+\left|\delta_{1}\right|\right) \vee & \left\{2-\left(\left|\alpha_{2}\right|+\left|\beta_{2}\right|\right)^{2}\right\} \\
& -\left(\left|\gamma_{2}\right|+\left|\delta_{2}\right|\right) \vee\left\{2-\left(\left|\alpha_{1}\right|+\left|\beta_{1}\right|\right)^{2}\right\}-\left(\left|\gamma_{1}\right|+\left|\delta_{1}\right|\right)\left(\left|\gamma_{2}\right|+\left|\delta_{2}\right|\right) \\
> & \left.(1.355)^{2}-2 \vee v 2-(1.355)^{2}\right\} \cdot(K-1.355)-(K-1.355)^{2}>1.59
\end{aligned}
$$


which contradicts $\left\|\mu^{m+n}\right\| \leqslant K$. Thus, without loss of generality, we may assume that only $u$ appears with the largest coefficient.

Let $\tilde{\mu}$ be the measure on $\mathbf{Z}_{2}$ obtained by taking the quotient by $\{e, u\}$. i.e.

$$
\tilde{\mu}=(\alpha+\beta) \tilde{e}+(\gamma+\delta) \tilde{v} .
$$

Since $\tilde{\mu}$ has bounded powers we must have $\tilde{\mu}=\lambda(\cos \theta \tilde{e}+i \sin \theta \tilde{v})$ for some $\theta$ and $|\lambda|=1$.

By Lemma $5, \gamma+\delta \neq 0$ and so $\theta \neq 0$ or $\pi$. Thus some power of $\tilde{\mu}$ has coefficient of $\tilde{v}$ with modulus $>0.866$. Let $\tilde{\mu}^{n}$ be the power and put

$$
\mu^{n}=\alpha e+\beta u+\gamma v+\delta u v \text {. }
$$

Then, we have $|\gamma+\delta|>0.866$ and so $|\alpha|+|\beta|<0.72026$. But one of these is the largest coefficient in $\mu^{n}$. By Lemma 1 (c) the second largest $>0.6$. This is necessarily one of $\gamma$ or $\delta$, but this would make $v$ or $u v$ have the largest coefficient in $\mu^{2 n}$. This is a contradiction.

We now return to the general case. Similar estimates to those in Lemma 6 can be obtained, but they are not good enough to give the result directly. We can manage with much cruder estimates to obtain that only a finite number of elements of order 2 occur with largest coefficients in the powers of $\mu$. Let $S=\{u \in G: u$ has the largest coefficient in $\mu^{ \pm 2^{n}}$ for some $\left.n\right\}$. Then by our assumption on $\mu, e \in S$. We will show that each nontrivial element in $S$ has order 2. For $u \in S$, let $N$ be the first positive integer such that $u$ has the largest coefficient in $\mu^{2^{N}}$. (If $n$ occurs in a negative power of $\mu$, a similar argument works.) By induction, we can assume that the largest coefficient in $\mu^{2^{N-1}}$ is that of $e$ or some $v \in S$ with $v^{2}=e$. In the case where $e$ has the largest coefficient, we can apply Theorem 2 to $\mu^{2^{N-1}}$ and obtain $u^{2}=e$. In the case where $v$ has the largest coefficient, we can apply Theorem 2 to $v^{-1} \mu^{2^{N-1}}$. Since $u$ has the largest coefficient of $\mu^{2^{N}}=v^{-2} \mu^{2^{N}}=\left(v^{-1} \mu^{2^{N-1}}\right)^{2}$, we obtain that $u^{2}=e$. In order to prove that $S$ is finite we need the following lemma.

LEMMA 9. If $\mu=\alpha e+\beta u+f$ and $\mu^{2}=\alpha_{1} u+\beta_{1} e+g$ where $|\alpha|$ and $\left|\alpha_{1}\right|$ are the largest coefficients in $\mu$ and $\mu^{2}$ respectively, and $\mu^{-1}=\mu^{*},\|\mu\|<K,\left\|\mu^{2}\right\|<K$ either $|\alpha|+|\beta| \geqslant \frac{1}{2}(K+1)$ or $\left|\alpha_{1}\right|+\left|\beta_{1}\right| \geqslant \frac{1}{2}(K+1)$.

Proof. Suppose that $|\alpha|+|\beta|<\frac{1}{2}(K+1)$. By Theorem $2,|\beta|$ is the second largest coefficient and $u^{2}=e$. By Lemma 4,

$$
|\alpha| \geqslant \frac{1}{4}\{K+1+\sqrt{ }(K+1)(5-3 K)\} .
$$

Hence $|\beta| \leqslant \frac{1}{2}(K+1)-|\alpha| \leqslant \frac{1}{4}\{K+1-\sqrt{ }(K+1)(5-3 K)\}$. Therefore

$$
\begin{aligned}
\left|\alpha_{1}\right| & \leqslant 2|\alpha \beta|+1-|\alpha|^{2}-|\beta|^{2}=1-(|\alpha|-|\beta|)^{2} \\
& \leqslant 1-\frac{1}{4}(5-3 K)(K+1) .
\end{aligned}
$$

Now if $\left|\alpha_{1}\right|+\left|\beta_{1}\right|<(K+1) / 2$, we can apply Lemma 4 to $\mu^{2}$ once we know that $\left|\beta_{1}\right|$ is the second largest coefficient. But

$$
\begin{aligned}
\left|\beta_{1}\right| & \geqslant|\alpha|^{2}-\left(1-|\alpha|^{2}\right)=2|\alpha|^{2}-1 \\
& \geqslant \frac{1}{8}(K+1)(6-2 K)+\frac{1}{4}(K+1) \vee(K+1)(5-3 K)-1 \\
& =\frac{1}{4}(K+1) \vee(K+1)(5-3 K)-\frac{1}{4}(K-1)^{2}>0.424,
\end{aligned}
$$


since $K<\frac{1}{3}(4 \cos (\pi / 9)+1)$. By Lemma $1(f)\left|\beta_{1}\right|$ is the second largest coefficient. Thus, by Lemma 4

$$
\left|\alpha_{1}\right| \geqslant \frac{1}{4}(K+1+\sqrt{ }(K+1)(5-3 K)) .
$$

From (1) and (2):

$$
\frac{1}{4}(K+1+\sqrt{ }(K+1)(5-3 K)) \leqslant 1-\frac{1}{4}(5-3 K)(K+1)
$$

i.e.

$$
\begin{aligned}
\vee(K+1)(5-3 K) & \leqslant 4-K-1-(5-3 K)(K+1) \\
& =3 K^{2}-3 K-2 .
\end{aligned}
$$

Therefore $(K+1)(5-3 K) \leqslant\left(3 K^{2}-3 K-2\right)^{2}$ which reduces to

$$
(K-1)\left(9 K^{3}-9 K^{2}-9 K+1\right) \geqslant 0 \text {. }
$$

But this is a contradiction since $K<\frac{1}{3}(4 \cos (\pi / 9)+1)$ which is a root of $9 \theta^{3}-$ $9 \theta^{2}-9 \theta+1=0$. Thus either $|\alpha|+|\beta|>\frac{1}{2}(K+1)$ or $\left|\alpha_{1}\right|+\left|\beta_{1}\right|>\frac{1}{2}(K+1)$.

Now, by continuity, and the fact that

$$
(K-1)\left(9 K^{3}-9 K^{2}-9 K+1\right)<0
$$

we have

COROllary 10. There exists $\varepsilon>0$ (depending only on $K$ ) such that either $|\alpha|+|\beta|>\frac{1}{2}(K+1)+\varepsilon$ or $\left|\alpha_{1}\right|+\left|\beta_{1}\right|>\frac{1}{2}(K+1)+\varepsilon$.

\section{LEMMA 11. $S$ is finite.}

Proof. Choose $\varepsilon>0$ as in Corollary 10. For $u, v \in S$, choose powers of $\mu$ such that

$$
\begin{array}{r}
\mu^{m}=\alpha_{1} e+\beta_{1} u+f \quad \text { with }\left|\alpha_{1}\right|+\left|\beta_{1}\right|>\frac{1}{2}(K+1)+\varepsilon, \\
\mu^{n}=\alpha_{2} e+\beta_{2} v+g \quad \text { with }\left|\alpha_{2}\right|+\left|\beta_{2}\right|>\frac{1}{2}(K+1)+\varepsilon .
\end{array}
$$

(Here, it is not assumed that $\left|\alpha_{i}\right| \geqslant\left|\beta_{i}\right|$-simply that $\left|\alpha_{i}\right|$ and $\left|\beta_{i}\right|$ are the two largest coefficients.) Let $\mu^{m}=\alpha_{1} e+\beta_{1} u+\gamma_{1} v+\delta_{1} u v+f_{1}$ and $\mu^{n}=\alpha_{2} e+\beta_{2} v+\gamma_{2} u$ $+\delta_{2} u v+g_{1}$. Now

$$
\|f * g\| \leqslant\left(K-\left|\alpha_{1}\right|-\left|\beta_{1}\right|\right)\left(K-\left|\alpha_{2}\right|-\left|\beta_{2}\right|\right)<\left[\frac{1}{2}(K-1)\right]^{2} .
$$

Also

$$
\begin{aligned}
\mu^{m+n}-f * g= & \left(\alpha_{1} e+\beta_{1} u\right)\left(\alpha_{2} e+\beta_{2} v\right)+\left(\alpha_{1} e+\beta_{1} u\right) * f+\left(\alpha_{2} e+\beta_{2} v\right) * g \\
= & \left(\alpha_{1} \alpha_{2}+\beta_{1} \gamma_{2}+\beta_{2} \gamma_{1}\right) e+\left(\alpha_{1} \beta_{2}+\alpha_{2} \gamma_{1}+\beta_{1} \delta_{2}\right) v \\
& +\left(\alpha_{2} \beta_{1}+\alpha_{1} \gamma_{2}+\beta_{2} \delta_{1}\right) u+\left(\beta_{1} \beta_{2}+\alpha_{2} \delta_{1}+\alpha_{1} \delta_{2}\right) u v+h
\end{aligned}
$$

where support of $h$ is disjoint from $\{e, u, v, u v\}$. Therefore

$$
\begin{aligned}
\left\|\mu^{m+n}\right\| \geqslant & \left|\alpha_{1} \alpha_{2}+\beta_{1} \gamma_{2}+\beta_{2} \gamma_{1}\right|+\left|\alpha_{1} \beta_{2}+\alpha_{2} \gamma_{1}+\beta_{1} \delta_{2}\right| \\
& +\left|\alpha_{2} \beta_{1}+\alpha_{1} \gamma_{2}+\beta_{2} \delta_{1}\right|+\left|\beta_{1} \beta_{2}+\alpha_{2} \delta_{1}+\alpha_{1} \delta_{2}\right|-\|f * g\| \\
\geqslant & \left(\left|\alpha_{1}\right|+\left|\beta_{1}\right|\right)\left(\left|\alpha_{2}\right|+\left|\beta_{2}\right|\right)-\left(\left|\alpha_{1}\right|+\left|\beta_{1}\right|\right)\left(\left|\gamma_{2}\right|+\left|\delta_{2}\right|\right) \\
& -\left(\left|\alpha_{2}\right|+\left|\beta_{2}\right|\right)\left(\left|\gamma_{1}\right|+\left|\delta_{1}\right|\right)-\|f * g\| .
\end{aligned}
$$


Since $\left\|\mu^{m+n}\right\| \leqslant K$, we have

$$
K \geqslant\left[\frac{1}{2}(K+1)+\varepsilon\right]^{2}-\frac{1}{2}(K+1)\left[\left|\gamma_{1}\right|+\left|\gamma_{2}\right|+\left|\delta_{1}\right|+\left|\delta_{2}\right|\right]-\left[\frac{1}{2}(K-1)\right]^{2} .
$$

Therefore

$$
\begin{aligned}
\left|\gamma_{1}\right|+\left|\gamma_{2}\right|+\left|\delta_{1}\right|+\left|\delta_{2}\right| & \geqslant\left[\frac{1}{2}(K+1)+\varepsilon\right]^{2}-\left[\frac{1}{2}(K-1)\right]^{2}-K \\
& =\varepsilon(K+1)+\varepsilon^{2}>\varepsilon(K+1) .
\end{aligned}
$$

This amount depends only on $K$. For each $u \in S$, choose a power $\mu^{n_{4}}$ of $\mu$ as above, and set $S_{u}=\left\{v \in S\right.$ : either $v$ or $u v$ has coefficient in $\mu^{n_{u}}$ with modulus $\left.>\frac{1}{4} \varepsilon(K+1)\right\}$. Clearly there is a bound for the cardinality of $S_{u}$ e.g. $4 K / \varepsilon(K+1)$, and the above has shown that if $u, v \in S$, then either $u \in S_{v}$ or $v \in S_{u}$. It follows that $S$ is finite.

REMARK. Because of Lemma 9, it would appear that Lemma 11 is tight at $K=\frac{1}{3}(4 \cos (\pi / 9)+1)$, but this is not so. In the proof, we have used the inequality

$$
(K-|\alpha|-|\beta|)^{2} \geqslant 1-|\alpha|^{2}-|\beta|^{2} \text {. }
$$

Now equality here means that $\mu$ is supported on only three elements, which is impossible if one of the elements is of order 2 and $|\alpha| \neq|\beta|$ (cf. Lemma 6).

We can now prove the main theorem for discrete groups.

THEOREM 4. If $\mu \in l_{1}(G)$ and has its powers norm-bounded by

$$
K<\frac{1}{3}(4 \cos (\pi / 9)+1)
$$

then $\mu$ has the form

$$
\mu=\lambda(\cos \theta x+i \sin \theta x u)
$$

where $u^{2}=e$ and $|\lambda|=1$.

Proof. We may assume that $e$ has the largest coefficient in $\mu$. Let $S=\{u \in G$ : $u$ has the largest coefficient in $\mu^{2^{n}}$ for some $n$ \}. Then $S$ is finite and consists of elements of order 2 . Let $H$ be the group generated by $S$. Then $H$ is the direct product of cyclic groups of order 2. Let $\phi: G \rightarrow G / H$ be the canonical map and $\tilde{\phi}$ the induced map of $l_{1}(G) \rightarrow l_{1}(G / H)$. Let $\tilde{\mu}=\tilde{\phi} \mu$. We will show that $\tilde{e}=\phi e$ has the largest coefficient in $\tilde{\mu}^{2^{n}}$ for all $n$. Suppose not. Then there exists $n$ and a coset $H z \neq H$ such that

$$
\mu^{2^{n}}=\sum \alpha(x) x \quad \text { with }\left|\sum_{x \in H z} \alpha(x)\right| \text { the largest coefficient. }
$$

Therefore

$$
\left|\sum_{x \in H z} \alpha(x)\right| \geqslant\left|\sum_{x \in H} \alpha(x)\right| .
$$

But the element $u$ with largest coefficient of $\mu^{2^{n}}$ is in $H$. Let

$$
\mu^{2^{n}}=\alpha_{1} u+\beta_{1} v+f \text { with }\left|\alpha_{1}\right| \geqslant\left|\beta_{1}\right|>\text { coefficients of } f
$$

Then

$$
\sum_{x \in H z}|\alpha(x)| \geqslant\left|\alpha_{1}\right|-\sum_{\substack{x \in H \\ x \neq u}}|\alpha(x)|
$$


i.e.

$$
\left|\alpha_{1}\right| \leqslant \sum_{x \in H z}|\alpha(x)|+\sum_{\substack{x \in H \\ x \neq u}}|\alpha(x)| \leqslant\left\|\mu^{2^{n}}\right\|-\left|\alpha_{1}\right| .
$$

Therefore $\left|\alpha_{1}\right|<\frac{1}{2}\left\|\mu^{2^{n}}\right\| \leqslant \frac{1}{2} K<0.8$.

By Lemma 1(c), $\left|\beta_{1}\right|>0.55$ and $\left|\alpha_{1}\right|+\left|\beta_{1}\right| \geqslant 1.35$. But the coefficient of $u v$ in $\mu^{2^{n+1}}$ has modulus $\geqslant\left(\left|\alpha_{1}\right|+\left|\beta_{1}\right|\right)^{2}-1>(1.35)^{2}-1>0.82$. Thus it is the largest, and so $u v \in H$. Hence $v \in H$ and we have $\Sigma_{x \in H_{z}}|\alpha(x)|<K-1.35<0.23$, which contradicts the fact that $\left|\Sigma_{x \in H z} \alpha(x)\right|$ is the largest coefficient in $\tilde{\mu}^{2^{n}}$. Now $\tilde{\mu}$ certainly has powers norm-bounded by $K$ and so by Theorem $1 \tilde{\mu}=\lambda \tilde{e}$ with $|\lambda|=1$. We now 'pull back' the elements of $H$ in turn. Choose $u \in S$, and let $H_{1}$ be a subgroup of $H$ of index 2 with $u \notin H_{1}$. We will consider the image $\mu_{1}$ of $\mu$ under the canonical projection $G \rightarrow G / H_{1}$. (We will abuse notation slightly and use $u, v$, etc., for their images in $G / H_{1}$.) Let $\mu_{1}=\alpha e+\beta u+f$. Then, since taking the quotient by the subgroup $\{e, u\}$ reduces $\mu_{1}$ to a multiple of the identity, we have $|\alpha+\beta|=1$ and $f=(e-u) * \sum \gamma_{i} y_{i}$ where there is at most one $y_{i}$ in each coset of $\{e, u\}$. Then $\|f\|=2 \Sigma\left|\gamma_{i}\right|<K-|\alpha|-|\beta|<K-1$ and $\|f\|_{2}^{2}=2 \Sigma\left|\gamma_{i}\right|^{2}$ $<\frac{1}{2}\left(2 \Sigma\left|\gamma_{i}\right|\right)^{2}<\frac{1}{2}(K-1)^{2}$. Let $\mu_{1}^{2}=\alpha_{1} e+\beta_{1} u+g$. Then $g=2(\alpha e+\beta u) * f+$ $f * f$. Hence

$$
\begin{aligned}
\|g\| & \geqslant 2\|(\alpha e+\beta u) * f\|-\|f\|^{2} \\
& =2\left\|(\alpha e+\beta u) *(e-u) * \sum \gamma_{i} y_{i}\right\|-\|f\|^{2} \\
& =2|\alpha-\beta|\|f\|-\|f\|^{2} .
\end{aligned}
$$

But

$$
\begin{aligned}
|\alpha-\beta|^{2} & =2|\alpha|^{2}+2|\beta|^{2}-|\alpha+\beta|^{2}=2\left(1-\|f\|_{2}^{2}\right)-1 \\
& =1-2\|f\|_{2}^{2}>1-(K-1)^{2}=2 K-K^{2} .
\end{aligned}
$$

Therefore $\|g\|>M\|f\|$ where $M=2|\alpha-\beta|-\|f\|>2 \sqrt{ }\left(2 K-K^{2}\right)-(K-1)$ $>1$ since $K<\frac{8}{5}$. Repeating this, we obtain $\left\|\mu_{1}^{2^{n}}\right\|>M^{n}\|f\|$. Since the norms are bounded, $\|f\|=0$.

Thus $\mu_{1}=\alpha e+\beta u$ and since $\mu_{1}^{-1}=\mu_{1}^{*}$ we have $\mu_{1}=\lambda(\cos \theta e+i \sin \theta u)$ for some $\theta$ and some $|\lambda|=1$. We now repeat the process. Choose $v \in S \cap H_{1}$, and let $\mathrm{H}_{2}$ be a subgroup of $H_{1}$ of index 2 with $v \notin H_{2}$. The image of $\mu$ on $G / H_{2}$ will have the form

$$
\mu_{2}=\alpha e+\beta u+\gamma v+\delta u v+g
$$

where $|\alpha+\gamma|=\cos \theta,|\beta+\delta|=\sin \theta$ and $g=\frac{1}{2}(e-v) * g$. If we now quotient out either the element $u$ or the element $u v$, we will obtain a measure of the correct form by the same proof as above. In particular we must have $g=\frac{1}{2}(e-u) * g$ and $g=\frac{1}{2}(e-u v) * g$. But this means

$$
g=\frac{1}{8}(e-u) *(e-v) *(e-u v) * g=0 .
$$

Thus $\mu_{2}$ is supported on a Klein 4-group, and by Theorem 3, it must be supported on a subgroup of order 2 . Repeating the argument for each element of $S$, we obtain the result. In fact it shows that $S$ has at most two elements. 
We now prove the main result for locally compact abelian groups. We will return to the usual notation of $\delta_{x}$ for the point measure at the point $x \in G$.

THEOREM 5. Let $G$ be a locally compact abelian group and $\mu \in M(G)$ have its powers norm-bounded by $K<\frac{1}{3}(4 \cos (\pi / 9)+1)$. Then $\mu$ has the form

$$
\mu=\lambda\left(\cos \theta \delta_{x}+i \sin \theta \delta_{x u}\right)
$$

where $u^{2}=e$ and $|\lambda|=1$.

Proof. Let $P$ be the projection of $M(G)$ onto $l_{1}(G)$. Since $\mu$ is invertible, $P \mu \neq 0$ and since $\left(P \mu^{n}\right)=(P \mu)^{n}$ and $\|P\|=1, P \mu \in l_{1}(G)$ and has powers norm-bounded by $K$. By Theorem 4 ,

$$
P \mu=\lambda\left(\cos \theta \delta_{x}+i \sin \theta \delta_{x u}\right)
$$

with $u^{2}=e$ and $|\lambda|=1$. If $\theta=0$, we have $\mu=\lambda \delta_{x}+\mu_{1}$ and $\left\|\mu_{1}\right\|<K-1$. Also $\mu^{2}=\lambda^{2} \delta_{x^{2}}+\nu$ where $\nu=2 \lambda \delta_{x} * \mu_{1}+\mu_{1} * \mu_{1}$. Hence $\|\nu\|>2\left\|\mu_{1}\right\|-\left\|\mu_{1}\right\|^{2}>$ $(3-K)\left\|\mu_{1}\right\|$. Now by iterating, since $3-K>1$, $\left\|\mu_{1}\right\|=0$. If $\theta \neq 0$, we can quotient out the subgroup $\{e, u\}$ and use the above to obtain that

$$
\mu=P \mu+\mu_{1} \quad \text { where } \mu_{1}=\frac{1}{2}\left(\delta_{e}-\delta_{u}\right) * \mu_{1}
$$

(i.e. $\mu_{1}$ must disappear under the quotient map). Now, as in Theorem 4, if $\mu^{2}=P \mu^{2}+\mu_{2}$

$$
\begin{aligned}
\mu_{2} & =2(P \mu) * \mu_{1}+\mu_{1} * \mu_{1} \\
& =2 \lambda\left(\cos \theta \delta_{x}+i \sin \theta \delta_{x u}\right) * \frac{1}{2}\left(\delta_{e}-\delta_{u}\right) * \mu_{1}+\mu_{1} * \mu_{1} \\
& =\lambda e^{-i \theta}\left(\delta_{e}-\delta_{u}\right) * \mu_{1}+\mu_{1} * \mu_{1} .
\end{aligned}
$$

Therefore $\left\|\mu_{2}\right\| \geqslant 2\left\|\mu_{1}\right\|-\left\|\mu_{1}\right\|^{2} \geqslant(3-K)\left\|\mu_{1}\right\|$. As before $\left\|\mu_{1}\right\|=0$. This completes the theorem.

3. Isomorphism theorems. We can now prove the isomorphism theorems. These follow easily from the previous results in much the same manner as they do in [3]. We will only give the case of an isomorphism between group algebras, but clearly we can use the same ideas to characterize those homomorphisms that preserve the identities of the groups.

THEOREM 6. Let $G_{1}$ and $G_{2}$ be abelian groups and $T$ an algebra isomorphism of $l_{1}\left(G_{1}\right)$ onto $l_{1}\left(G_{2}\right)$ with $\|T\|<\frac{1}{3}(4 \cos (\pi / 9)+1)$. Then, either $G_{1}$ and $G_{2}$ are isomorphic or each has a subgroup of order $2, H_{1}$ and $H_{2}$ respectively, such that $G_{1} / H_{1}$ and $G_{2} / H_{2}$ are isomorphic. In fact we always have $\|T\| \leqslant \vee 2$.

(N.B. The form of these isomorphisms can be described explicitly. For $\|T\|<$ $\sqrt{ } 2$, this was given in [3]. The case $\|T\|=\sqrt{ } 2$ will be given in the next theorem.)

Proof. For each $x \in G_{1}, T x \in l_{1}\left(G_{2}\right)$ and has its powers norm-bounded by $K$. Thus by Theorem 4 , its powers are norm-bounded by $\sqrt{ } 2$ and it has the form

$$
T x=\lambda[\cos \theta t(x)+i \sin \theta t(x) u]
$$

where $|\lambda|=1$, and $t(x), u \in G_{2}, u^{2}=e$. 
Since this is true for all $x,\|T\| \leqslant \vee 2$. Now suppose that for each $x \in G_{1}, T x$ has only one element of $G_{2}$ in its support. Then

$$
T x=\lambda(x) t(x) \text { for all } x \in G_{1} .
$$

Clearly $\lambda \in \hat{G}_{1}$ and $t$ is an isomorphism of $G_{1}$ onto $G_{2}$. On the other hand, suppose that for some $x \in G_{1}, T x$ has two elements of $G_{2}$ in its support. Then

$$
T x=\lambda(x)[\cos \theta t(x)+i \sin \theta t(x) u]
$$

where $|\lambda(x)|=1, u, t(x) \in G_{2}, u^{2}=e$, and $\cos \theta \neq 0, \sin \theta \neq 0$. If

$$
T y=\lambda(y)[\cos \phi t(y)+i \sin \phi t(y) v]
$$

with $t(y), v \in G_{2}, v^{2}=e, \cos \phi \neq 0, \sin \phi \neq 0$, then clearly $u=v$, or else $T x y$ would not have the correct form-its support would contain four elements of $G_{2}$. Let $H_{2}=\{e, u\}$ and $\tilde{T}: l_{1}\left(G_{1}\right) \rightarrow l_{1}\left(G_{2} / H_{2}\right)$ be defined by $\tilde{T}=P T$ where $P$ is the canonical projection $l_{1}\left(G_{2}\right) \rightarrow l_{1}\left(G_{2} / H_{2}\right)$. Then $\tilde{T}$ has the form

$$
\tilde{T} x=\lambda(x) s(x)
$$

with $\lambda \in \hat{G}_{1}$ and $s$ a homomorphism of $G_{1}$ onto $G_{2} / H_{2}$. Now since $T$ is an isomorphism onto $l_{1}\left(G_{2}\right)$, there exists $v \in G_{1}$ such that $u$ belongs to the support of $T v$, i.e. $T v=\lambda\left[\cos \theta e_{2}+i \sin \theta u\right]$ with $\sin \theta \neq 0$. Hence

$$
T v^{2}=\lambda^{2}\left[\cos 2 \theta e_{2}+i \sin 2 \theta u\right]
$$

and so $T e_{1}, T v$ and $T v^{2}$ are linearly dependent. It follows that $e_{1}, v$ and $v^{2}$ are linearly dependent and so $v^{2}=e_{1}$. Thus $T v= \pm u$, and it follows that the kernel of $s$ is $\left\{e_{1}, v\right\}=H_{1}$ say. Now, by the isomorphism theorem for groups, $G_{1} / H_{1}$ is isomorphic to $G_{2} / H_{2}$, and the proof is complete.

The converse of this is also true. If $G_{1}$ and $G_{2}$ are abelian groups with subgroups $H_{1}$ and $H_{2}$ each of order 2, such that $G_{1} / H_{1}$ and $G_{2} / H_{2}$ are isomorphic, then there exists an algebra isomorphism from $l_{1}\left(G_{1}\right)$ onto $l_{1}\left(G_{2}\right)$ with norm $<\sqrt{ } 2$. For, let $K$ be the common quotient, $s: G_{1} \rightarrow K, t: G_{2} \rightarrow K$ be the projections, and $H_{1}=$ $\left\{e_{1}, v\right\}, H_{2}=\left\{e_{2}, u\right\}$. Choose $\phi \in \hat{G}_{1}$ with $\phi(v)=-1$, and $\psi \in \hat{G}_{2}$ with $\psi(u)=-1$. Define

$$
T x=\frac{1}{2}(1+\phi(x) \psi(y)) y+\frac{1}{2}(1-\phi(x) \psi(y)) y u
$$

where $s(x)=t(y)$, and extend $T$ to a map from $l_{1}\left(G_{1}\right)$ onto $l_{1}\left(G_{2}\right)$ by linearity. ( $T$ is well defined, since $\psi(u)=-1$.) It is easily checked that $T$ is an algebra isomorphism and that $\|T\| \leqslant \vee 2$. For the norm to equal $\vee 2$, there must exist $x \in G_{1}$ such that $\phi(x) \psi(y)$ is arbitrarily close to $\pm i$.

We now describe the form of all isomorphisms with norm $<\frac{1}{3}(4 \cos (\pi / 9)+1)$.

THEOREM 7. Let $T$ be an algebra isomorphism from $l_{1}\left(G_{1}\right)$ and $l_{1}\left(G_{2}\right)$ with $\|T\|<\frac{1}{3}(4 \cos (\pi / 9)+1)$.

(1) If $\|T\|<\frac{1}{2}(1+\sqrt{ } 3)$, then $\|T\|=1$ and $T$ has the form

$$
T x=\psi(x) s(x) \quad\left(x \in G_{1}\right)
$$

where $\psi \in \hat{G}_{1}$ and $s$ is an isomorphism of $G_{1}$ onto $G_{2}$. 
(2) If $\frac{1}{2}(1+\sqrt{ } 3) \leqslant\|T\|<\sqrt{ } 2$, then $\|T\|=\sqrt{ } 2 \cos (\pi / 4 n)$ for some odd number $n$, and $T$ has the form

$$
T x=\psi(x)\left\{\frac{1}{2}(1+\phi(x)) s(x)+\frac{1}{2}(1-\phi(x)) s(x) u\right\} \quad\left(x \in G_{1}\right)
$$

where $\phi, \psi \in \hat{G}_{1}, \phi$ has order $n, u \in G_{2}$ is of order 2 , and $s$ is an isomorphism of $G_{1}$ onto $G_{2}$.

(3) If $\sqrt{ } 2 \leqslant\|T\|<\frac{1}{3}(4 \cos (\pi / 9)+1)$, then $\|T\|=\sqrt{ } 2$ and $G_{1}$ and $G_{2}$ have subgroups $H_{1}=\left\{e_{1}, v\right\}$ and $H_{2}=\left\{e_{2}, u\right\}$ respectively such that $G_{1} / H_{1} \simeq G_{2} / H_{2} \cong$ $K$. If $s: G_{1} \rightarrow K, t: G_{2} \rightarrow K$ are the natural projections, then $T$ has the form

$$
T x=\lambda(x)\left\{\frac{1}{2}(1+\phi(x) \psi(y)) y+\frac{1}{2}(1-\phi(x) \psi(y)) y u\right\}
$$

$\left(x \in G_{1}, s(x)=t(y)\right)$, where $\lambda, \phi \in \hat{G}_{1}$ and $\psi \in \hat{G}_{2}$.

Proof. (1) and (2) are Theorem 2.6 of [3].

(3) By Theorem 6, we have $\|T\|=\sqrt{ } 2$, and that $G_{1} /\left\{e_{1}, v\right\}$ is isomorphic to $G_{2} /\left\{e_{2}, u\right\}$. Also $\tilde{T} x=\lambda(x) s(x)$, where $\lambda \in \hat{G}_{1}$ and $s$ is the natural projection $G_{1} \rightarrow K=G_{1} /\left\{e_{1}, v\right\}$. If $s(x)=\{y, y u\}$, we can write

$$
T x=\lambda(x)\left\{\frac{1}{2}(1+\gamma(x, y)) y+\frac{1}{2}(1-\gamma(x, y)) y u\right\}
$$

which defines a function $\gamma$ on the set $A=\left\{(x, y) \in G_{1} \times G_{2}: s(x)=t(y)\right\}$. (Here $t$ is the natural projection $G_{2} \rightarrow K$.) $\gamma$ clearly satisfies $\gamma(x, u y)=-\gamma(x, y),(x, y) \in$ $A$. Since $T v=\lambda(v) u, \gamma(x v, y)=-\gamma(x, y),(x, y) \in A$. Now $A$ is a subgroup of $G_{1} \times G_{2}$, and since $T$ is an isomorphism, it is routine to show that $\gamma$ is a character on $A$. By [2,24.12], $\gamma$ admits an extension to a character on $G_{1} \times G_{2}$, which may be written in the form $\gamma(x, y)=\phi(x) \psi(y)$ where $\phi \in \hat{G}_{1}$ and $\psi \in \hat{G}_{2}$. This gives the required form.

COROLlaRY 8. If $G_{1}$ and $G_{2}$ are abelian, without elements of order 2, and if $T$ : $l_{1}\left(G_{1}\right) \rightarrow l_{1}\left(G_{2}\right)$ is an algebra isomorphism with $\|T\|<\frac{1}{3}(4 \cos (\pi / 9)+1)$ then $G_{1}$ and $G_{2}$ are isomorphic.

This is the best possible result as the following example shows.

EXAMPLE. Consider the algebra isomorphism from $l_{1}\left(\mathbf{Z}_{9}\right) \rightarrow l_{1}\left(\mathbf{Z}_{3} \times \mathbf{Z}_{3}\right)$ defined by

$$
T x=\frac{1}{3}\left(1+2 \cos \frac{2 \pi}{9}\right) v+\frac{1}{3}\left(1+2 \cos \frac{8 \pi}{9}\right) v u+\frac{1}{3}\left(1+2 \cos \frac{14 \pi}{9}\right) v u^{2}
$$

where $x$ is a generator of $\mathbf{Z}_{9}$, and $u, v$ are the generators of $\mathbf{Z}_{3} \times \mathbf{Z}_{3}$. Then as we remarked earlier $T x$ has its powers norm bounded by $\frac{1}{3}(4 \cos (\pi / 9)+1)$. Thus $\|T\|=\frac{1}{3}(4 \cos (\pi / 9)+1)$.

The results for topological groups follow in much the same way as they did in [2]. For the measure algebras, we simply restrict attention to the discrete measures and use Theorem 6. The continuity properties follow from results of Greenleaf in [1]. For the $L^{1}$-algebras, we again use results of Greenleaf in [1] to extend the isomorphism to one between the measure algebras. 
THEOREM 9. Let $G_{1}$ and $G_{2}$ be locally compact abelian groups and $T: L^{1}\left(G_{1}\right) \rightarrow$ $L^{1}\left(G_{2}\right)\left(M\left(G_{1}\right) \rightarrow M\left(G_{2}\right)\right)$ be an algebra isomorphism with $\|T\|<\frac{1}{3}(4 \cos (\pi / 9)+1)$. Then, either $G_{1}$ and $G_{2}$ are isomorphic, or each has a subgroup of order 2 and their quotients are isomorphic.

(The form of these isomorphisms is clear from Theorem 7.)

THEOREM 10. Let $G_{1}$ and $G_{2}$ be compact abelian groups and $T: C\left(G_{1}\right) \rightarrow C\left(G_{2}\right)$ $\left[L^{\infty}\left(G_{1}\right) \rightarrow L^{\infty}\left(G_{2}\right)\right]$ be an algebra isomorphism with $\|T\|<\frac{1}{3}(4 \cos (\pi / 9)+1)$. Then, either $G_{1}$ and $G_{2}$ are isomorphic, or each has a subgroup of order 2 and their quotients are isomorphic.

(Again the forms of these isomorphisms may be described explicitly.)

I would like to thank the referee for his careful reading of the manuscript, and for his helpful comments and suggestions.

\section{REFERENCES}

1. F. P. Greenleaf, Norm-decreasing homomorphisms of group algebras, Pacific J. Math. 15 (1965), $1187-1219$.

2. E. Hewitt and K. A. Ross, Abstract harmonic analysis. Vol. I, Springer-Verlag, Berlin and New York, 1963.

3. N. J. Kalton and G. V. Wood, Homomorphisms of group algebras with norm less than V2, Pacific J. Math. 62 (1976), 439-460.

4. W. Rudin, Fourier analysis on groups, Interscience, New York, 1960.

5. B. M. Schreiber, Measures with bounded convolution powers, Trans. Amer. Math. Soc. 151 (1970), 405-431.

Department of Pure Mathematics, University College of Swansea, Singleton Park, Swansea SA2 8PP, Wales, Great Britian 